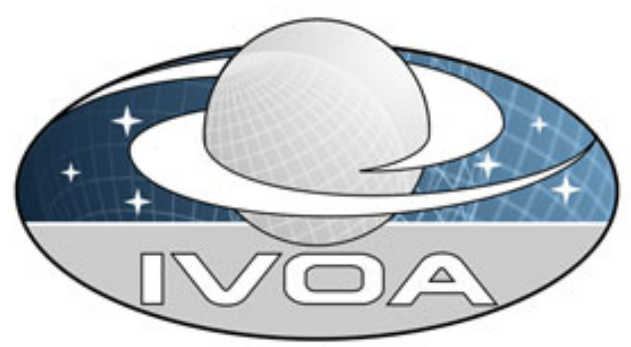

\title{
Describing Simple Data Access Services Version 1.0
}

\section{IVOA Recommendation 5 October 2013}

\section{This version:}

\section{Latest version:}

http://www.ivoa.net/Documents/SimpleDALRegExt/20131005/

http://www.ivoa.net/Documents/SimpleDALRegExt/

\section{Previous versions:}

PR: http://www.ivoa.net/Documents/SimpleDALRegExt/20130911/

PR: http://www.ivoa.net/Documents/SimpleDALRegExt/20121116/

PR: http://www.ivoa.net/Documents/SimpleDALRegExt/20120517/

WD: http://www.ivoa.net/Documents/SimpleDALRegExt/20110921/

\section{Authors:}

\author{
Raymond Plante, Editor, \\ Jesus Delago, \\ Paul Harrison, \\ Doug Tody, \\ and the IVOA Registry Working Group
}

\section{Abstract}

An application that queries or consumes descriptions of $\mathrm{VO}$ resources must be able to recognize a resource's support for standard IVOA protocols. This specification describes how to describe a service that supports any of the four fundamental data access protocols--Simple Cone Search (SCS), Simple Image Access (SIA), Simple Spectral Access (SSA), Simple Line Access (SLA)--using the VOResource XML encoding standard. A key part of this specification is the set of VOResource XML extension schemas that define new metadata that are specific to those protocols. This document describes in particular rules for describing such services within the context of IVOA Registries and data discovery as well as the VO Standard Interface (VOSI) and service self-description. In particular, this document spells out the essential mark-up needed to identify support for a standard protocol and the base URL required to access the interface that supports that protocol.

\section{Status of this document}

This document has been produced by the IVOA Registry Working Group.

It has been reviewed by IVOA Members and other interested parties, and has been endorsed by the IVOA Executive Committee as an IVOA Recommendation as of 5 October 2013. It is a stable document and may be used as reference material or cited as a normative reference from another document. IVOA's role in 
making the Recommendation is to draw attention to the specification and to promote its widespread deployment. This enhances the functionality and interoperability inside the Astronomical Community.

Early versions of this document were known as RegSimpleDAL. The short name is now SimpleDALRegExt.

A list of current IVOA Recommendations and other technical documents can be found at http://www.ivoa.net/Documents/.

\section{Acknowledgements}

This document has been developed with support from the National Science Foundation's Information Technology Research Program under Cooperative Agreement AST0122449 with The Johns Hopkins University, from the UK Particle Physics and Astronomy Research Council (PPARC), and from the Eurpean Commission's Sixth Framework Program via the Optical Infrared Coordination Network (OPTICON).

\section{Conformance-related definitions}

The words "MUST", "SHALL", "SHOULD", "MAY", "RECOMMENDED", and "OPTIONAL" (in upper or lower case) used in this document are to be interpreted as described in IETF standard, RFC 2119 [RFC 2119].

The Virtual Observatory (VO) is general term for a collection of federated resources that can be used to conduct astronomical research, education, and outreach. The International Virtual Observatory Alliance (IVOA) is a global collaboration of separately funded projects to develop standards and infrastructure that enable VO applications.

$\mathrm{XML}$ document validation is a software process that checks that an XML document is not only well-formed $\mathrm{XML}$ but also conforms to the syntax rules defined by the applicable schema. Typically, when the schema is defined by one or more XML Schema [schema] documents (see next section), validation refers to checking for conformance to the syntax described in those Schema documents. This document describes additional syntax constraints that cannot be enforced solely by the rules of XML Schema; thus, in this document, use of the term validation includes the extra checks that goes beyond common Schema-aware parsers which ensure conformance with this document.

\section{Syntax Notation Using XML Schema}

The Extensible Markup Language, or XML, is document syntax for marking textual information with named tags and is defined by the World Wide Web Consortium (W3C) Recommendation, XML 1.0 [XML]. The set of $\mathrm{XML}$ tag names and the syntax rules for their use is referred to as the document schema. One way to formally define a schema for XML documents is using the W3C standard known as XML Schema [schema].

This document defines the VOResource schema using XML Schema. The full Schema document is listed in Appendix A. Parts of the schema appear within the main sections of this document; however, documentation nodes have been left out for the sake of brevity.

Reference to specific elements and types defined in the VOResource schema include the namespaces prefix, vr, as in vr: Resource (a type defined in the VOResource schema). Reference to specific elements and types defined in the VODataService extension schema include the namespaces prefix, vs, as in vs : ParamHTTP (a type defined in the VODataService schema). Use of the vs prefix in compliant instance documents is strongly recommended, particularly in the applications that involve IVOA Registries (see [RI], section 3.1.2). Elsewhere, the use is not required. 


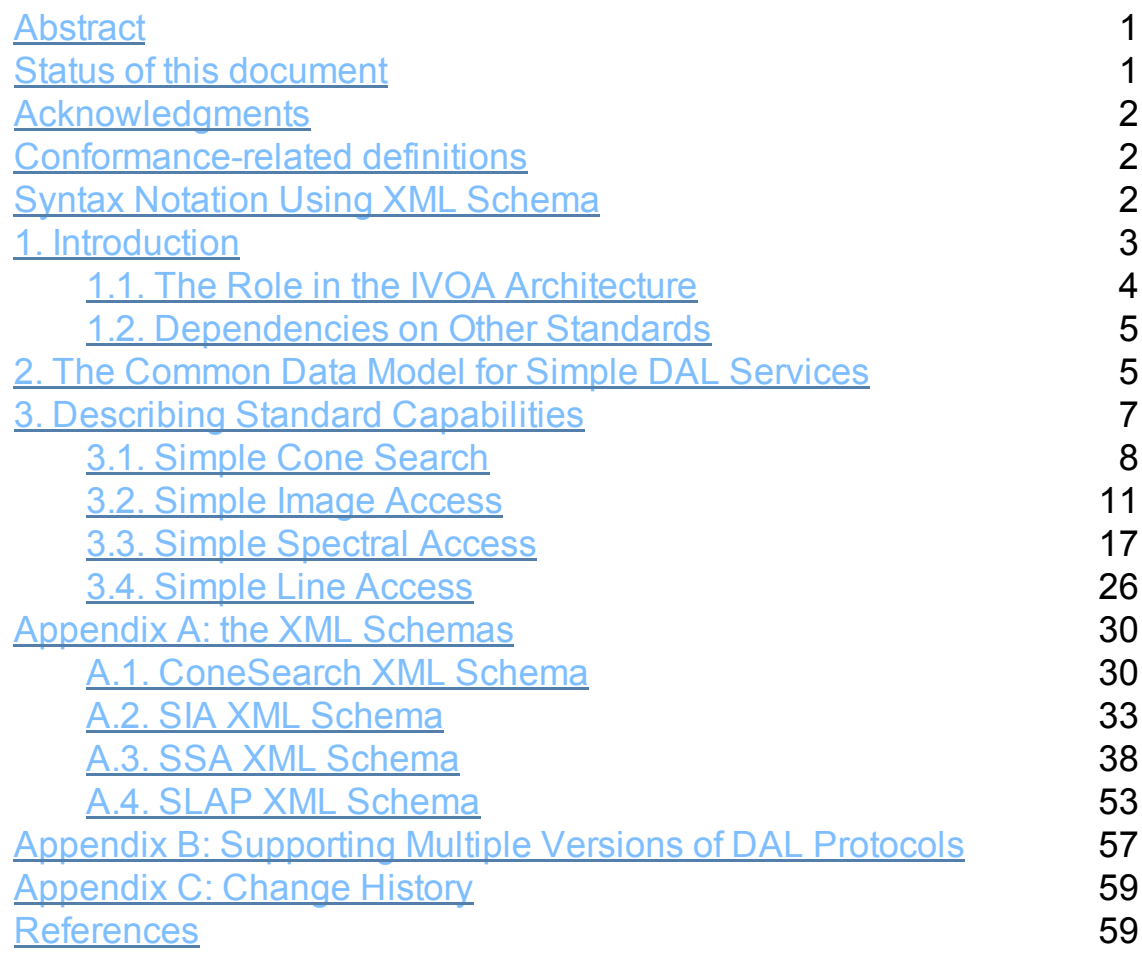

\section{Introduction}

Four data access service protocols play a key role in discovering data in the VO:

- Simple Cone Search [SCS] -- searches a catalog for sources or observations that are within a given distance of a sky position.

- Simple Image Access $[\underline{S \mid A]}$-- searches an archive for images that overlap a given region of sky.

- Simple Spectral Access [SSA] -- searches an archive for spectra of positions within a given region of sky.

- Simple Line Access $[\underline{S L A}]$-- searches a catalog specializing in descriptions of spectral line transitions.

They are called "simple" because a typical query can be formed using only a few search parameters encoded into a URL (i.e. an HTTP GET request). Their power for data discovery comes from the ability of an application to form a single query according to the rules of one of these protocols and send it to multiple services selected, say, for their relevence to a scientific topic which support that protocol. The results collected from those services, in effect then, represent all the relevent data of that type known to the VO. Thus, the key for an application wishing to do a comprehensive search of the VO is to discover all of the services that support the particular standard protocol.

Service discovery in the VO is done via a searchable registry [RI]-i.e., a searchable repository of descriptions of resources in VO. These descriptions are comprised of common standard metadata [RM] that capture information about what a resource contains or does and who provides it. A standard registry encodes these descriptions using the VOResource XML Schema [VOR]. Service resources in particular include capability metadata that describe the functionality it supports along with interface metadata that describe how to access that functionality. It is within the capability metadata that it is possible to indicate support for a particular standard protocol.

Capability metadata play an important role beyond just identifying support for a standard interface. More generally, they describe how the service behaves, and if applications are to make use of this information in an automated way, the behavior must be described using standardized metadata. In general, the metadata necessary for describing that behavior will be specific to the particular kind of service. In the case of a 
standard protocol, in which it is common that some variation in behavior is allowed while still being in compliance, it can be important to an application to know how a service complies with the standard for two reasons:

1. The application may wish to search for and select services that support a particular protocol feature. For example, an application may wish to find image services that can create cut-outs on-the-fly.

2. The application may wish to plan its use of the service according its limitations, such as the maximum region of sky that can be searched in one query.

It is important to note that the relevent behavioral differences between separate services that support a common protocol--and thus the metadata used to describe those behaviors--will be specific to that protocol. That is, for example, the ability to create image cut-outs is irrelevent to the Simple Cone Search protocol. Consequently, it is necessary to define protocol-specific metadata to adequately describe a service's support for that protocol. This document defines such capability metadata for SCS, SIA, SSA, and SLA.

This document describes for each of the standard data access protocols--SCS, SIA, SSA, and SLA-precisely how to describe a service that supports one of the protocols in terms of the VOResource XML encoding standard [VOR]. This specification is intended to be applicable wherever VOResource records are used, but in particular, it is intended as the standard for encoding resource descriptions within an IVOAcompliant registry $[\mathrm{R}]$ ] and for encoding capability metadata available through the VO Standard Interface [VOSI].

\subsection{The Role in IVOA Architecture}

The IVOA Architecture [Arch] provides a high-level view of how IVOA standards work together to connect users and applications with providers of data and services, as depicted in the diagram in Fig. 1.

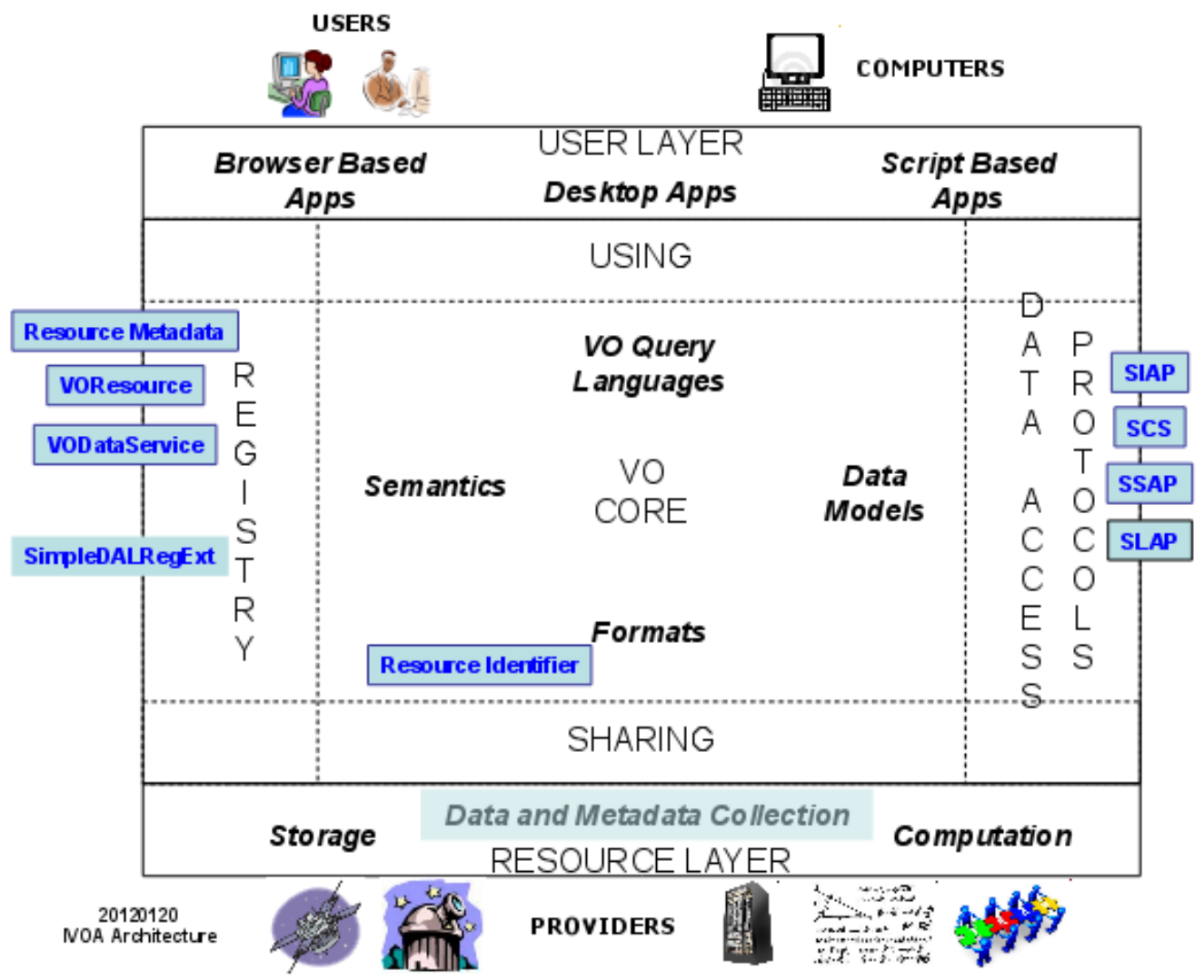

Figure 1. SimpleDALRegExt in the IVOA Architecture. The Registry enables applications in the User Layer to discover archives in the Resource Layer and the services they provide for accessing data, particularly those that support the standard data access protocols like SIAP, SCS, SSAP, and SLAP (illustrated on the right). The registry metadata model standards (in blue text and boxes on the left) give structure to the information that enables that discovery. In particular, the SimpleDALRegExt standard defines the metadata used to describe standard data access services of the types listed on the right. 
In this architecture, data access protocols provide the means for users (via the User Layer) to access data from archives. Of particular importance are the standard protocols, SCS, SIA, SSA, and SLA, which allow a generic user tool to find data in any archive that supports those protocols. Registries provide to tools in the User Layer a means to discover which archives support the standard protocols. A registry is a repository of descriptions of resources, such as standard services, that can be searched based on the metadata in those descriptions.

Resource descriptions have a well-defined structure: the core concepts are defined in the Resource Metadata standard [RM], and the format is defined by the VOResource XML standard [VOR]. Additional metadata specialized to describe a specific kind of service are defined via extensions to the VOResource core XML Schema. SimpleDALRegExt is one such extension specifically for describing SCS, SIA, SSA, and SLA services in the registry.

\subsection{Dependencies on Other Standards}

This specification relies directly on other IVOA standards in the following ways:

\section{VOResource, v1.03 [VOR]:}

Descriptions of services that support the standard protocols are encoded using the VOResource XML Schema. The protocol-specific schemas defined in this document are extensions of the VOResource core schema.

Simple Cone Search, v1.03 [SCS],

Simple Image Access, v1.0 [SIA],

Simple Spectral Access, v1.04 [SSA],

Simple Line Access, v1.0 [SLA]:

Each protocol specification describes the metadata concepts that should be included in a description of a service that supports the specification.

\section{VODataService, v1.1 [VDS]:}

The interface to the standard protocol functionality is described with a specialized Interface type, vs : ParamHTTP, which is defined in the VODataService XML Schema, an extension to VOResource. This document also recommends describing the service using VODataService resource type, vs: CatalogDataService.

Except where noted in subsequent sections, this specification does not imply support for any other versions (including later versions) than the ones noted above.

This specification refers to other IVOA standards:

\section{Registry Interfaces, v1.0 [RI]:}

A registry that is compliant with both this specification and the Registry Interfaces standard will encode service resource descriptions according to the recommendations in this document.

\section{IVOA Standard Interface, v1.0 [VOSI]:}

A service that supports one of the target protocols as well as the capability metadata retrieval method defined by VOSI [VOSI, section 2.1] is compliant with this specifcation if the capability metadata are encoded according the recommendations in this document.

Unlike with the previously mentioned specifications, this specification may apply to later versions of the RI and VOSI standards.

\section{The Common Data Model for Simple DAL Services}

This section describes common requirements for describing the target DAL services as VOResource records.

To be recognized as a service, the DAL service resource must be described as a resource type of 
$v r$ : Service (defined in the VOResource schema [VOR]) or one of its legal sub-types. As specified in the VOResource specification [VOR], the resource type is set by setting the xsi: type attribute on the element representing the root of the VOResource record to the namespace-qualified resource type name. As the DAL services respond to queries with tables of available data products, the resource should set the resource type to vs: CatalogService (defined in the VODataService extension schema [VDS]). In this case, record authors are encouraged to include a full description of the columns in the table returned in query response (assuming full verbosity). The vs : CatalogService resource type also allows the record to provide sky coverage information which authors are also encouraged to provide; an exception to this would be for pure SLA services as the spectral line catalogs they serve are not strictly sky observations.

Note:

In the future, a more appropriate resource type may be defined for describing DAL services; thus, the loose requirement on the resource type allows for this. At the time of this writing, the vs: CatalogService is recommended as the most appropriate type and represents current practice with IVOA registries.

The VOResource record must include a <capability> element that describes the services support for the DAL protocol. The contents of the element is described in the next section. In all cases, the value of the <capability> element's standardID unambiguously identifies the service's support for the particular DAL protocol. The resource may include other <capability> elements to describe support for other protocols.

The <capability> element describing support for the DAL protocol must include a child <interface> element that describes support for the required DAL interface; the xsi: type attribute on that element must be set to vs : ParamHTTP, and the role attribute must be set to "std". A <accessURL> element within that <interface > must be set to the base URL, as defined in the DAL protocol specification, that provides access to the standard DAL protocol. It is not necessary to provide the use attribute to the <accessURL> element (as its value can be assumed); however, when it is provided, it must be set to "base". Similarly, it is not necessary to provide the <interface> element with <queryType> or <resultType> elements; however, when provided, their values should be "GET" and "application/x-votable+xml", respectively. The vs : ParamHTTP allows one to describe input parameters supported by the service; description authors are encouraged to list the optional parameters and any custom parameters supported by the instance of the service.

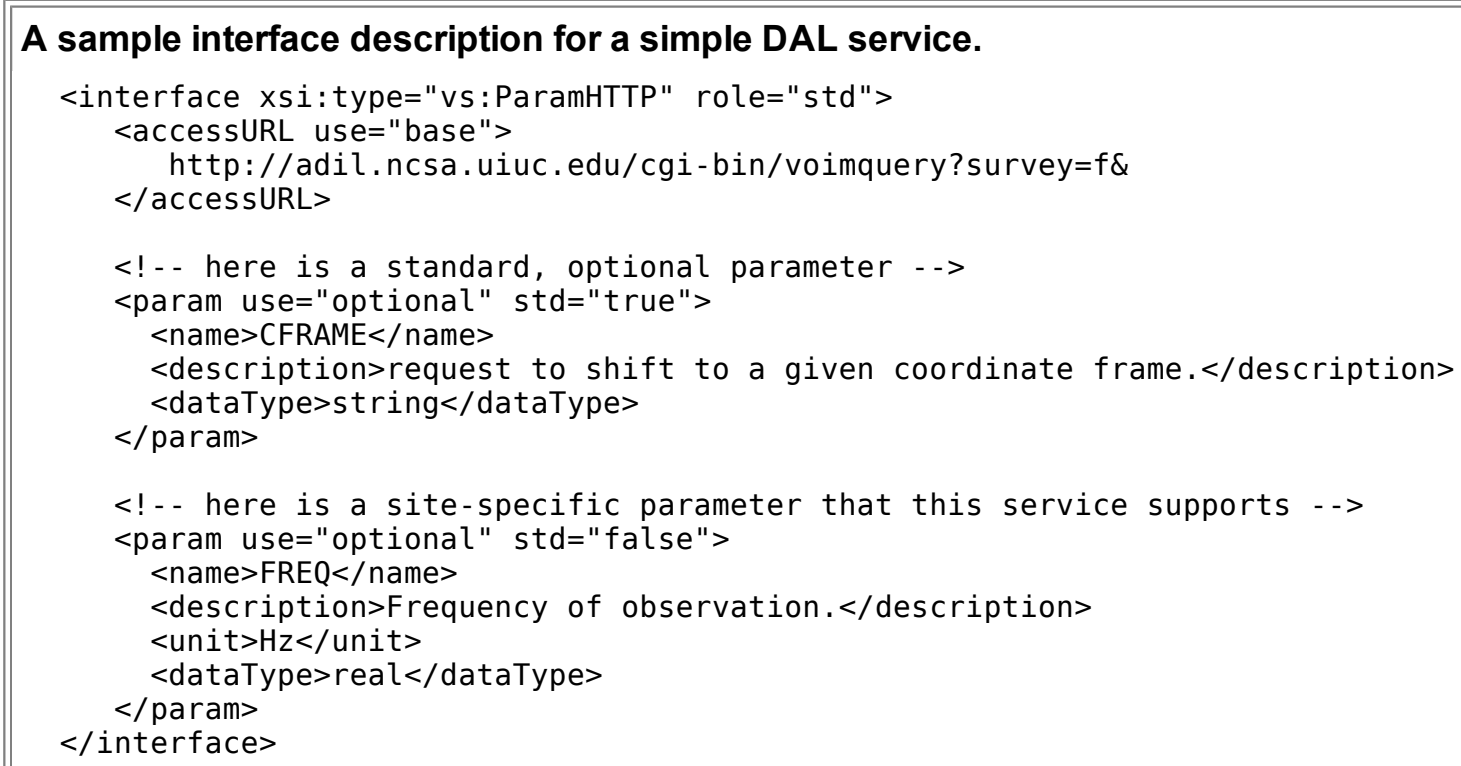




\section{Describing Standard Capabilities}

This section describes the specific VOResource metadata extension schemas used to describe support for the target DAL protocols. The purpose of these schemas are to provide the vr: Capability sub-type that identifies the specific protocol. These are defined employing the recommendations for vr: Capability extensions given in the VOResource standard [VR]. In particular, each extension schema has the following features:

- The namespace associated with the extension is a URI that is intended to resolve an HTTP URL to $\mathrm{XML}$ Schema document that defines the extension schema. This means that VOResource document authors may use this URI as the location URL within the value of xsi: schemaLocation attribute.

Note:

The IVOA Registry Interface standard $[\mathrm{R} \mid]$ actually requires that the VOResource records it shares with other registries provide location URLs via xsi: schemaLocation for the VOResource schema and all legal extension schemas that are used in the records. This rule would apply to the extension schemas defined in this standard.

- A particular namespace prefix is recommended for use when referring to the specialized vr: Capability sub-type defined in the schema. Generally instance documents may use any prefix; however, in applications where common and consistent use of prefixes is recommended (such as with the Registry Interface specification [R]], use of the prefixes recommended in this document can be used.

- The schema sets elementFormDefault="unqualified". This means that it is not necessary to qualify element names defined in the schema with a namespace prefix (as there are no global elements defined). The only place it is usually needed is as a qualifier to a Capability sub-type name given as the value of an xsi: type attribute on the <capability> element (see the examples in the subsections below).

- The definition of the specialized vr: Capability sub-type fixes the value of its standardID attribute to the URI that is intended to uniquely identify the standard DAL protocol whose support the type describes.

- The specialized vr: Capability sub-type includes a <testQuery> element for encoding parameters that together can be used to test the service. The format for encoding the individual parameters is customized for each of the four simple services covered in this specification.

Note:

It is also possible to encode test queries as part of the vs: ParamHTTP interface via its <testQuery> element [VDS]; this query is encoded as a single string that can be appended to the service's base URL. This specification does not recommend one over the other. Use of the vr: Capability-based <testQuery> pre-dates the vs : ParamHTTP-based one and has been kept for backward-compatibility purposes. It might be easier for some clients to use since each parameter is individually tagged and perhaps easier to parse, manipulate, and enter automatically into an interface. The vs: ParamHTTP-based one has an advantage in that it can be applied to any REST-like service, standard or not. 


\subsection{Simple Cone Search}

This section describes the ConeSearch VOResource metadata extension schema which is used to describe services that comply with the Simple Cone Search protocol [SCS].

\subsubsection{The Schema Namespace}

The namespace associated with the ConeSearch extension schema is "http://www.ivoa.net/xml/ConeSearch/v1.0". The namespace prefix, cs, should be used in applications where common use of prefixes improves interoperability (e.g. in the IVOA registries [RI]). Furthermore, we use the cs prefix in this document to refer to types defined as part of the ConeSearch extension schema.

\subsubsection{ConeSearch}

The cs: ConeSearch type is a vr: Capability sub-type that should be used to describe a service's support for the Simple Cone Search protocol [SCS]; it is defined as follows:

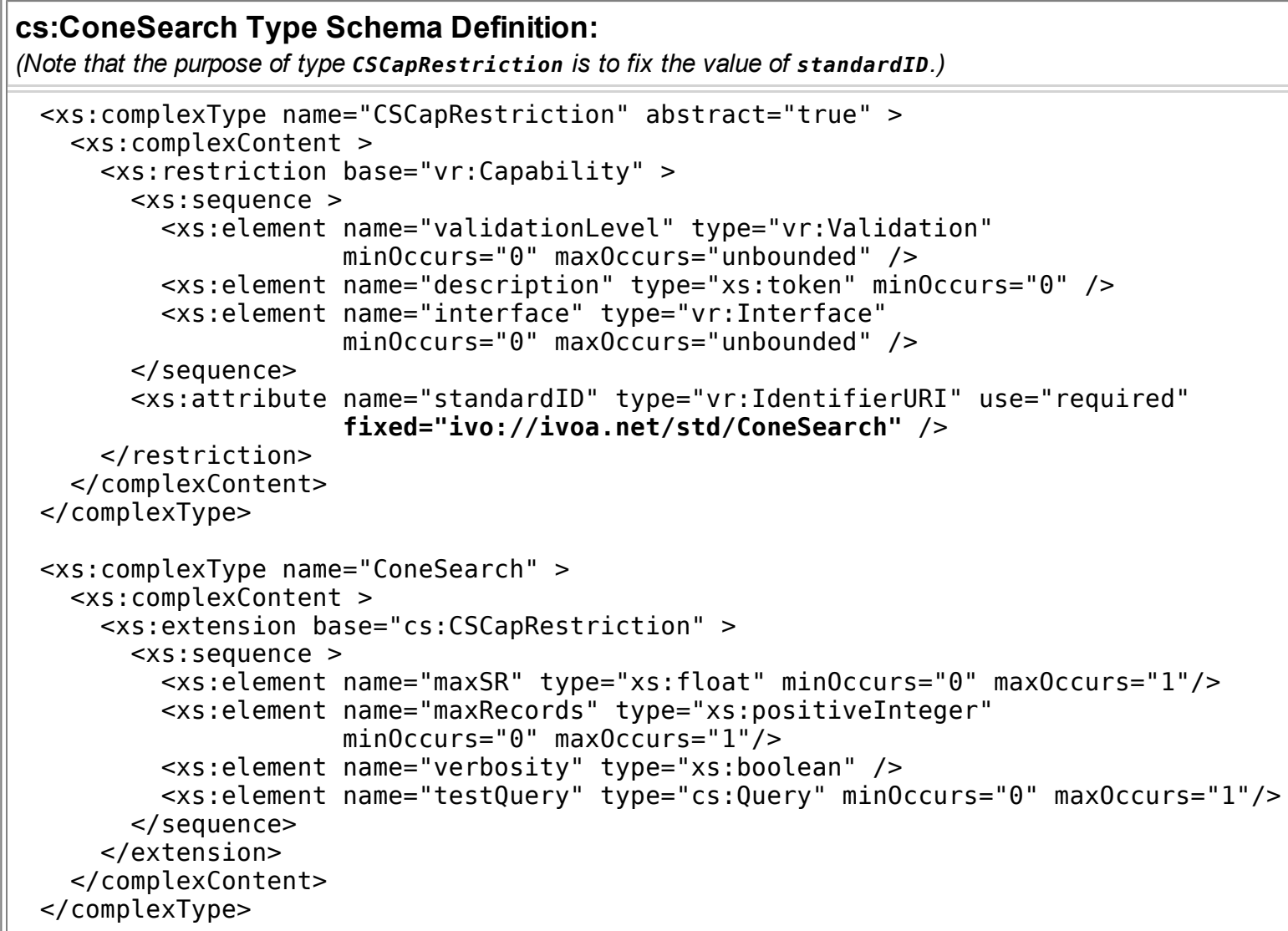

The cs: CSCapRestriction is defined to fix the value of the standardID attribute; thus, all uses of the cs : ConeSearch type must set standardID to "ivo://ivoa.net/std/ConeSearch". Because the cs: CSCapRestriction is marked as abstract, instance documents can not use it directly as a value for the xsi : type attribute.

The custom metadata that the cs: ConeSearch type provides is given in the table below. For the elements whose semantics map directly to service profile metadata called for in the SCS standard [SCS, section 3], there is an entry labeled "SCS Name"; this indicates the metadata name given in the SCS specification that the element in this schema corresponds to. The profile metadata listed in the SCS specification that is not covered by the elements below are covered by other metadata that are part of the core VOResource schema. 


\begin{tabular}{|c|c|c|}
\hline \multicolumn{3}{|c|}{ cs:ConeSearch Extension Metadata Elements } \\
\hline Element & & Definition \\
\hline $\operatorname{maxSR}$ & $\begin{array}{l}\text { SCS Name: } \\
\text { Value type: } \\
\text { Semantic Meaning: } \\
\text { Occurrences: } \\
\text { Comments: }\end{array}$ & $\begin{array}{l}\text { MaxSR } \\
\text { floating-point number: xs : float } \\
\text { : The largest search radius, in degrees, that will be accepted by the } \\
\text { service without returning an error condition. Not providing this element } \\
\text { or specifying a value of } 180 \text { indicates that there is no restriction. } \\
\text { optional } \\
\text { Not providing a value is the prefered way to indicate that there is no } \\
\text { restriction. }\end{array}$ \\
\hline maxRecords & \begin{tabular}{|l} 
SCS Name: \\
Value type: \\
Semantic Meaning: \\
Occurrences: \\
Comments:
\end{tabular} & $\begin{array}{l}\text { MaxRecords } \\
\text { positive integer: xs : positiveInteger } \\
\text { The largest number of records that the service will return. Not providing } \\
\text { this value means that there is no effective limit. } \\
\text { optional } \\
\text { This does not refer to the total number of records in the catalog but } \\
\text { rather maximum number of records the service is capable of returning. A } \\
\text { limit that is greater than the number of records available in the archive is } \\
\text { equivalent to their being no effective limit. (See RM, Hanisch } 2007 .)\end{array}$ \\
\hline verbosity & $\begin{array}{l}\text { SCS Name: } \\
\text { Value type: } \\
\text { Semantic Meaning: } \\
\text { Occurrences: } \\
\text { Comments: }\end{array}$ & $\begin{array}{l}\text { Verbosity } \\
\text { boolean (true/false): xs : boolean } \\
\text { True if the service supports the VERB keyword; false, otherwise. } \\
\text { required } \\
\text { The value should be false if the all values of the VERB input parameter } \\
\text { results in the same set of columns being returned. }\end{array}$ \\
\hline testQuery & $\begin{array}{l}\text { Value type: } \\
\text { Semantic Meaning: } \\
\text { Occurrences: }\end{array}$ & $\begin{array}{l}\text { composite: } \mathrm{cs:Query} \\
\text { A query that will result in at least one matched record that can be used } \\
\text { to test the service. } \\
\text { optional }\end{array}$ \\
\hline
\end{tabular}

\subsubsection{1 testQuery and the Query Type}

The <testQuery> element is intended to help other VO components (e.g. registries, validation services, services that monitor the VO's operational health--but typically not end users) test that the service is up and operating correctly. It provides a set of legal input parameters that should return a legal response that includes at least one matched record. Since this query is intended for testing purposes, the size of the result set should be small.

The cs : Query type captures the different components of the query into separate elements, as defined below: 


\section{cs:Query Type Schema Definition}

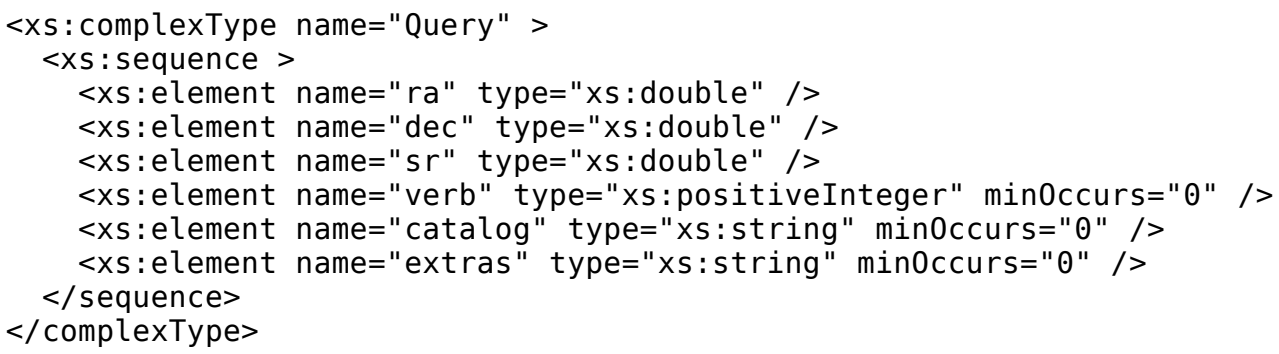

The individual sub-elements are defined as follows:

\begin{tabular}{|c|c|}
\hline \multicolumn{2}{|c|}{ cs:Query Metadata Elements } \\
\hline Element & Definition \\
\hline ra & $\begin{array}{l}\text { Value type: } \quad \text { floating-point number: xs : double } \\
\text { Semantic Meaning: the right ascension of the search cone's center in decimal degrees. } \\
\text { Occurrences: } \quad \text { required }\end{array}$ \\
\hline dec & $\begin{array}{l}\text { Value type: } \quad \text { floating-point number: xs : double } \\
\text { Semantic Meaning: the declination of the search cone's center in decimal degrees. } \\
\text { Occurrences: } \quad \text { required }\end{array}$ \\
\hline sr & $\begin{array}{l}\text { Value type: } \quad \text { floating-point number: xs : double } \\
\text { Semantic Meaning: the radius of the search cone in decimal degrees. } \\
\text { Occurrences: } \quad \text { required }\end{array}$ \\
\hline verb & 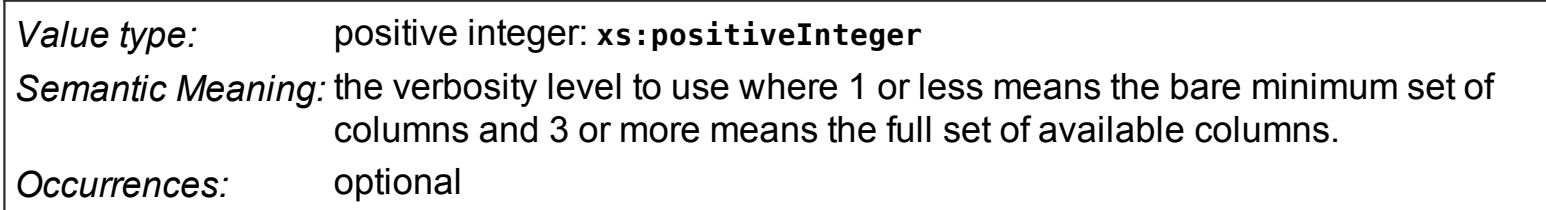 \\
\hline catalog & $\begin{array}{ll}\text { Value type: } & \text { string: xs: string } \\
\text { Semantic Meaning: the catalog to query. } \\
\text { Occurrences: } \quad \text { optional } \\
\text { Comments: } & \text { When the service can access more than one catalog, this input parameter, if } \\
& \text { available, is used to indicate which service to access. }\end{array}$ \\
\hline extras & 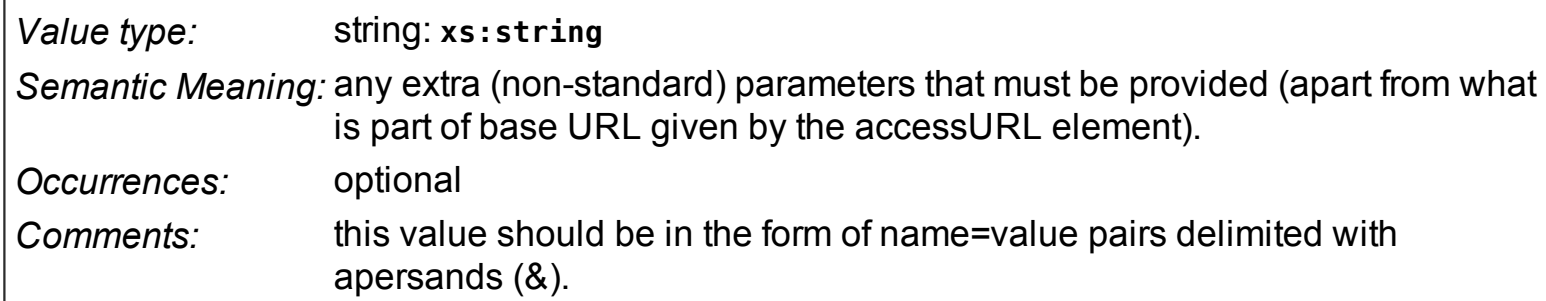 \\
\hline
\end{tabular}




\subsection{Simple Image Access}

This section describes the SIA VOResource metadata extension schema which is used to describe services that comply with the Simple Image Access protocol [SIA].

\subsubsection{The Schema Namespace}

The namespace associated with the SIA extension schema is "http://www.ivoa.net/xml/SIA/v1.1". The namespace prefix, sia, should be used in applications where common use of prefixes improves interoperability (e.g. in the IVOA registries [RI]). Furthermore, we use the sia prefix in this document to refer to types defined as part of the SIA extension schema.

\subsubsection{SimplelmageAccess}

The sia: SimpleImageAccess type is a vr: Capability sub-type that should be used to describe a service's support for the Simple Image Access protocol [SIA]; it is defined as follows:

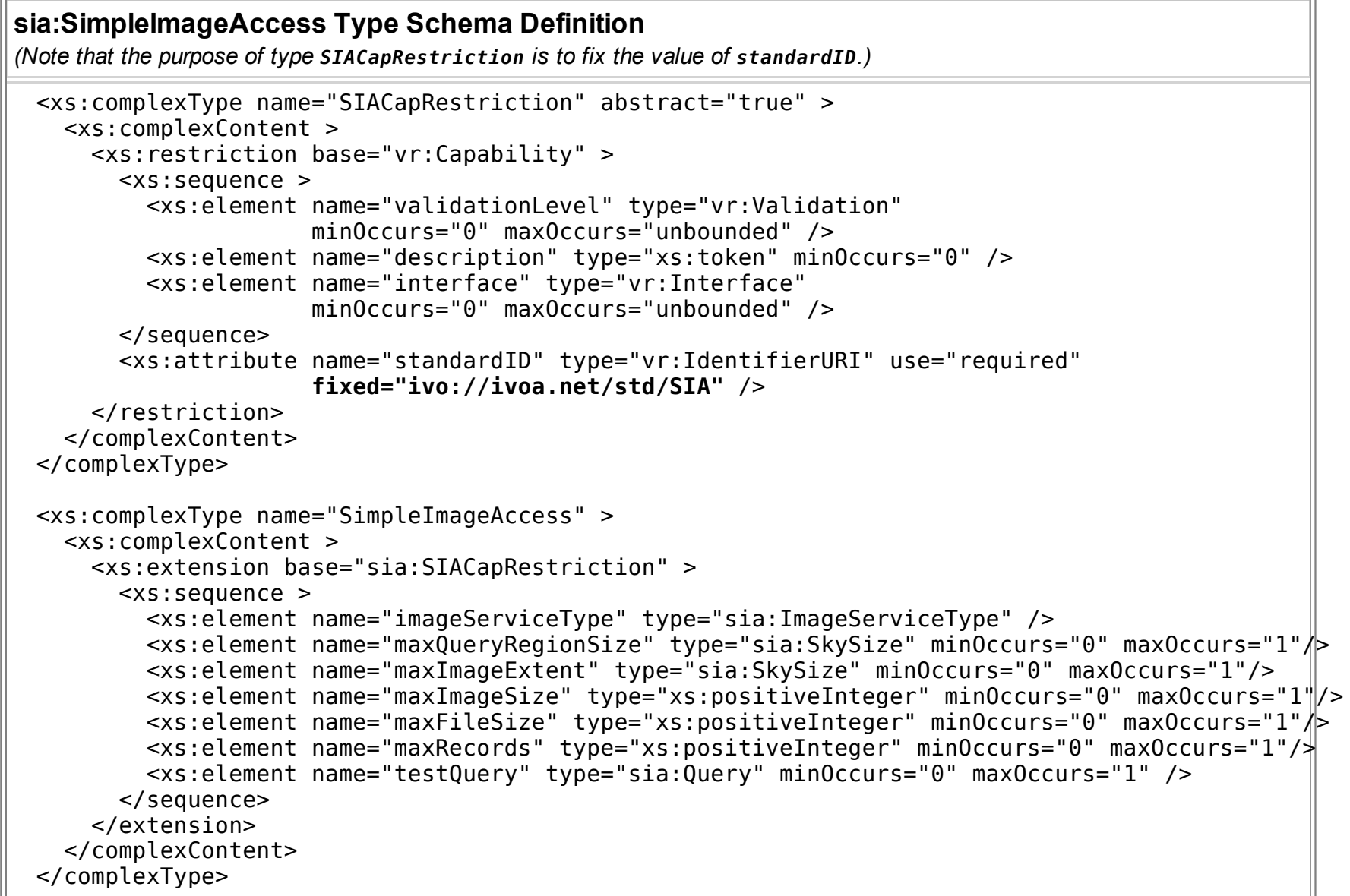

The sia:SIACapRestriction is defined to fix the value of the standardID attribute; thus, all uses of the sia: SimpleImageAccess type must set standardID to "ivo://ivoa.net/std/SIA". Because the sia: SIACapRestriction is marked as abstract, instance documents can not use it directly as a value for the xsi : type attribute.

The custom metadata that the sia: SimpleImageAccess type provides is given in the table below. For the elements whose semantics map directly to metadata called for in the SIA standard [SIA, section 7], there is an entry labeled "SIA Name"; this indicates the metadata name given in the SIA specification that the element in this schema corresponds to. 


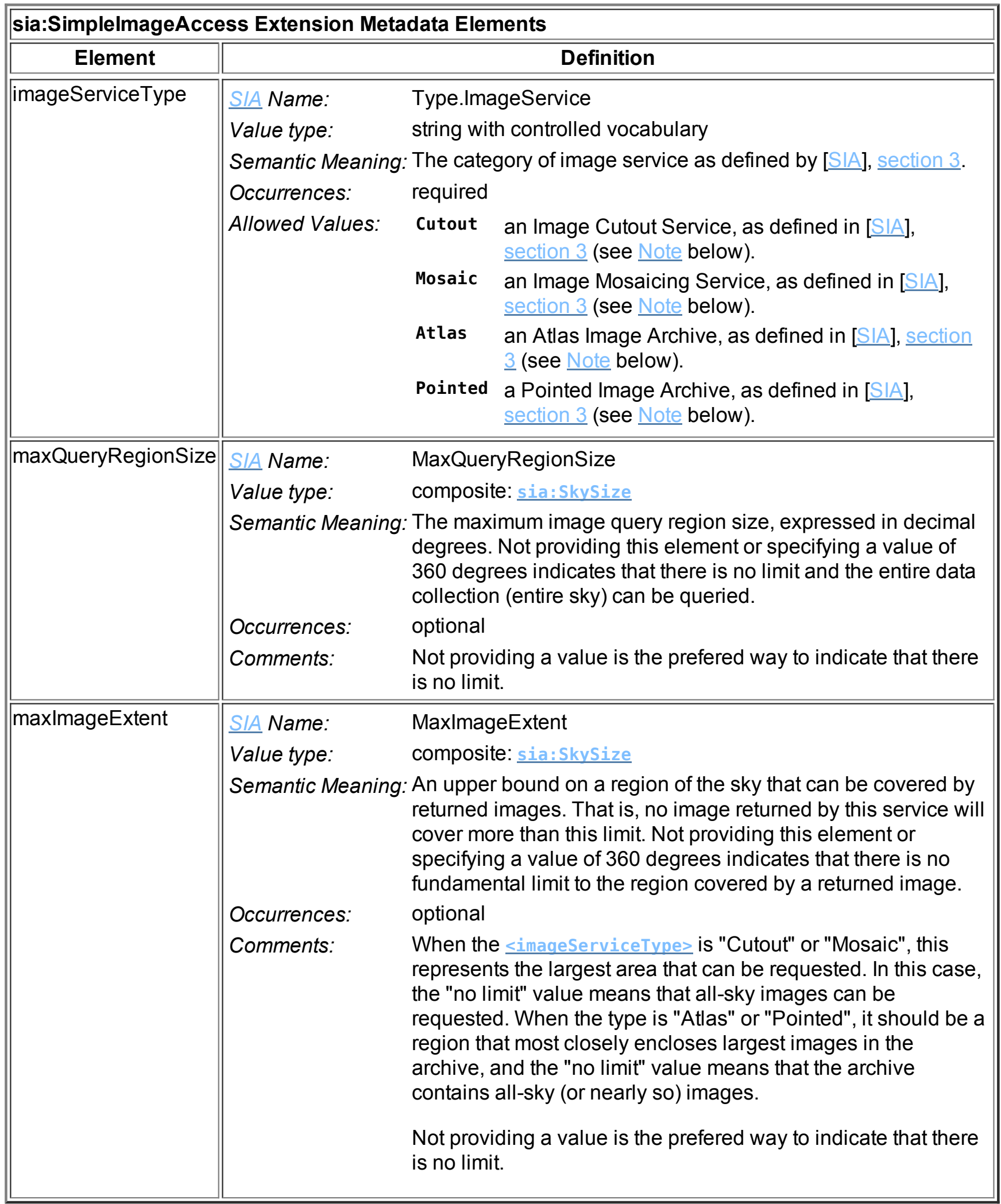




\begin{tabular}{|c|c|c|}
\hline \multicolumn{3}{|c|}{ sia:SimplelmageAccess Extension Metadata Elem ents (con't) } \\
\hline Element & & Definition \\
\hline maxlmageSize & $\begin{array}{l}\text { SIA Name: } \\
\text { Value type: } \\
\text { Semantic Meaning: } \\
\text { Occurrences: } \\
\text { Comments: } \\
\end{array}$ & $\begin{array}{l}\text { MaxImageSize } \\
\text { positive integer: xs : positiveInteger } \\
\text { A measure of the largest image the service can produce given as the } \\
\text { maximum number of pixels along the first or second axes. Not } \\
\text { providing a value indicates that there is no effective limit to the size of } \\
\text { the images that can be returned. } \\
\text { optional } \\
\text { This is primarily relevant when the } \leq \text { imageServiceType }>\text { is "Cutout" or } \\
\text { "Mosaic", indicating the largest image that can be created. When the } \\
\leq \text { imageServiceType }>\text { is "Atlas" or "Pointed", this should be specified } \\
\text { only when there are static images in the archive that can be searched } \\
\text { for but not returned because they are too big. } \\
\text { When a service is more fundamentally limited by the total number of } \\
\text { pixels in the image, this value should be set to the square-root of that } \\
\text { number. This number will then represent a lower limit on the } \\
\text { maximum length of a side. }\end{array}$ \\
\hline maxFileSize & $\begin{array}{l}\text { SIA Name: } \\
\text { Value type: } \\
\text { Semantic Meaning: } \\
\text { Occurrences: } \\
\text { Comments: }\end{array}$ & $\begin{array}{l}\text { MaxFileSize } \\
\text { positive integer: xs : positiveInteger } \\
\text { The maximum image file size in bytes. Not providing a value indicates } \\
\text { that there is no effective limit the size of files that can be returned. } \\
\text { optional } \\
\text { This is primarily relevant when the } \leq \text { imageServiceType }>\text { is "Cutout" or } \\
\text { "Mosaic", indicating the largest files that can be created. When the } \\
\leq \text { imageServiceType }>\text { is "Atlas" or "Pointed", this should be specified } \\
\text { only when there are static images in the archive that can be searched } \\
\text { for but not returned because they are too big. }\end{array}$ \\
\hline maxRecords & $\begin{array}{l}\text { SIA Name: } \\
\text { Value type: } \\
\text { Semantic Meaning: } \\
\text { Occurrences: } \\
\text { Comments: }\end{array}$ & $\begin{array}{l}\text { MaxRecords } \\
\text { positive integer: xs : positiveInteger } \\
\text { The largest number of records that the Image Query web method will } \\
\text { return. Not providing this value means that there is no effective limit. } \\
\text { optional } \\
\text { This does not refer to the total number of images in the archive but } \\
\text { rather maximum number of records the service is capable of returning. } \\
\text { A limit that is greater than the number of images available in the } \\
\text { archive is equivalent to their being no effective limit. (See also [RM], } \\
\text { Sect. 5.2.) }\end{array}$ \\
\hline testQuery & $\begin{array}{l}\text { Value type: } \\
\text { Semantic Meaning: } \\
\text { Occurrences: }\end{array}$ & $\begin{array}{l}\text { composite: sia: Query } \\
\text { a set of query parameters that is expected to produce at least one } \\
\text { matched record which can be used to test the service. } \\
\text { optional }\end{array}$ \\
\hline
\end{tabular}

The sia: ImageServiceType type is provided to restrict the values of the <imageServiceType> element to those allowed by the SIA standard: 


\section{sia:ImageServiceType Type Schema Definition}

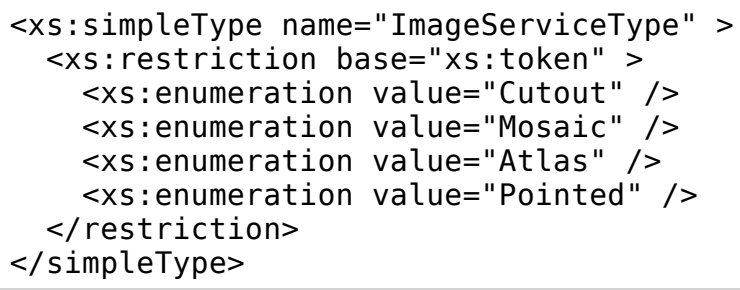

Note:

The SIA specification defines the image service types as follows:

\section{Image Cutout Service}

This is a service which extracts or "cuts out" rectangular regions of some larger image, returning an image of the requested size to the client. Such images are usually drawn from a database or a collection of survey images that cover some large portion of the sky. To be considered a cutout service, the returned image should closely approximate (or at least not exceed) the size of the requested region; however, a cutout service will not normally resample (rescale or reproject) the pixel data. A cutout service may mosaic image segments to cover a large region but is still considered a cutout service if it does not resample the data. Image cutout services are fast and avoid image degredation due to resampling.

\section{Image Mosaicing Service}

This service is similar to the image cutout service but adds the capability to compute an image of the size, scale, and projection specified by the client. Mosaic services include services which resample and reproject existing image data, as well as services which generate pixels from some more fundamental dataset, e.g., a high energy event list or a radio astronomy measurement set. Image mosaics can be expensive to generate for large regions but they make it easier for the client to overlay image data from different sources. Image mosaicing services which resample already pixelated data will degrade the data slightly, unlike the simpler cutout service which returns the data unchanged.

\section{Atlas Image Archive}

This category of service provides access to pre-computed images that make up a survey of some large portion of the sky. The service, however, is not capable of dynamically cutting out requested regions, and the size of atlas images is predetermined by the survey. Atlas images may range in size from small cutouts of extended objects to large calibrated survey data frames.

\section{Pointed Image Archive}

This category of service provides access to collections of images of many small, "pointed" regions of the sky. "Pointed" images normally focus on specific sources in the sky as opposed to being part of a sky survey. This type of service usually applies to instrumental archives from observatories with guest observer programs (e.g., the HST archive) and other general purpose image archives (e.g., the ADIL). If a service provides access to both survey and pointed images, then it should be considered a Pointed Image Archive for the purposes of this specification; if a differentiation between the types of data is desired the pointed and survey data collections should be registered as separate image services.

Several of the sia: SimpleImageAccess metadata use complex types to capture their values; the subsequent sections below define those special types. 


\subsubsection{SkySize}

The sia: SkySize type is used to capture simple rectangular extents on the sky along longitudinal and latitudinal directions. It is defined as follows:

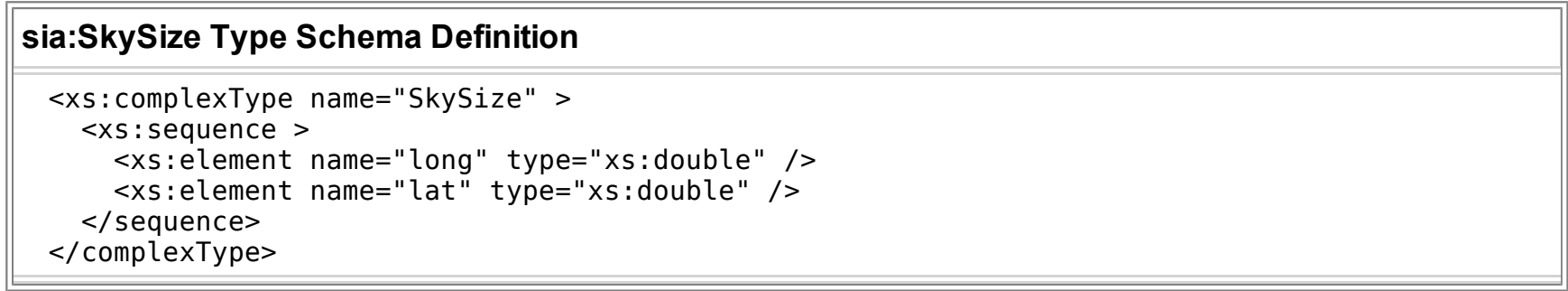

The coordinate system for the region is intended to be implied by the context of its use--i.e. the definition of the element defined with this type. In this SIA case, the longitudinal and latitudinal values represent the extents along right ascension and declination in the ICRS system (the system assumed by the SIA interface).

\begin{tabular}{|c|c|}
\hline sia:SkyS & ize Metadata Elements \\
\hline Element & Definition \\
\hline long & $\begin{array}{l}\text { Value type: } \quad \text { floating-point number: xs : double } \\
\text { Semantic Meaning: } \text { The maximum size in the longitude (R.A.) direction given in degrees } \\
\text { Occurrences: } \quad \text { required }\end{array}$ \\
\hline lat & $\begin{array}{l}\text { Value type: } \quad \text { floating-point number: xs : double } \\
\text { Semantic Meaning: } \text { The maximum size in the latitude (Dec.) direction given in degrees } \\
\text { Occurrences: } \quad \text { required }\end{array}$ \\
\hline
\end{tabular}

\subsubsection{3. testQuery and the Query Type}

As with the other DAL vr: capability types, the <testQuery> element is intended to help other VO components (e.g. registries, validation services, services that monitor the VO's operational health--but typically not end users) test that the service is up and operating correctly. It provides a set of legal input parameters that should return a legal response that includes at least matched record. Since this query is intended for testing purposes, the size of the result set should be small.

The sia: Query type captures the different components of the query into separate elements, as defined below:

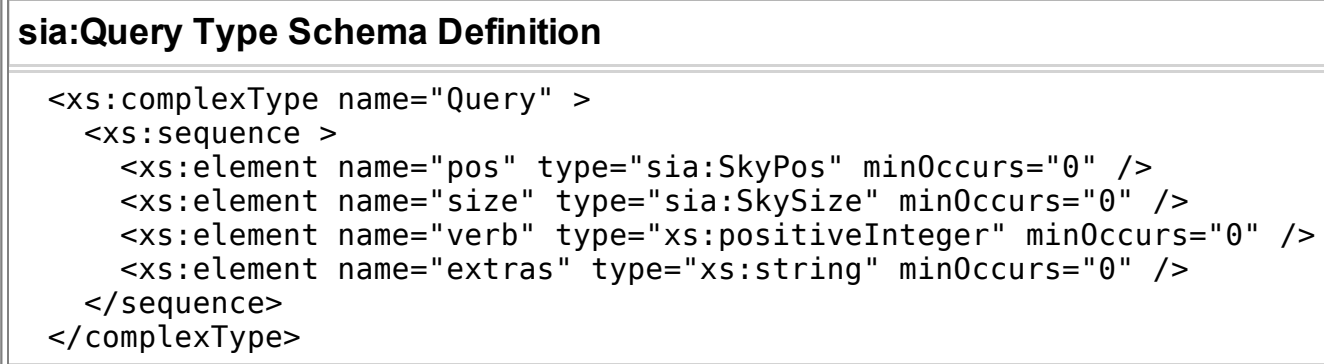


The individual sub-elements are defined as follows:

\begin{tabular}{|c|c|}
\hline \multicolumn{2}{|c|}{ sia:Query Metadata Elements } \\
\hline Element & Definition \\
\hline pos & 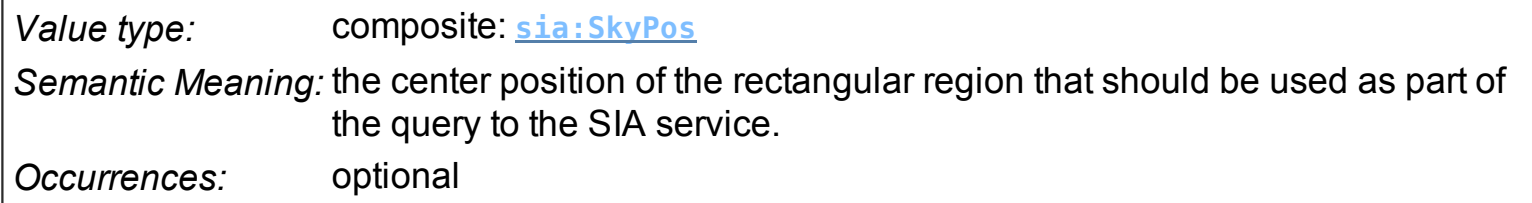 \\
\hline size & 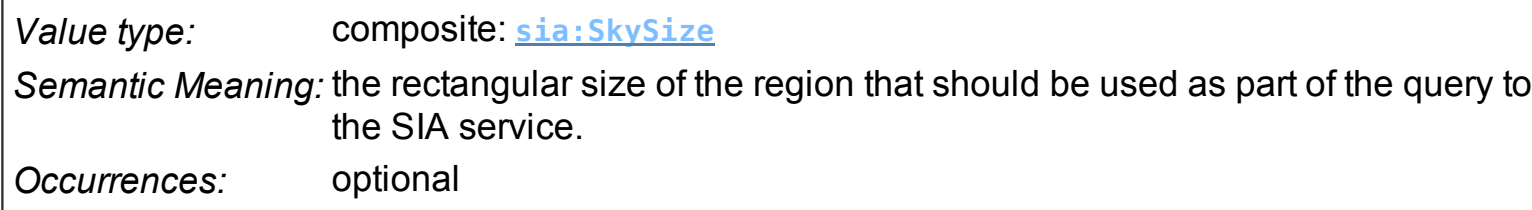 \\
\hline verb & $\begin{array}{l}\text { Value type: } \quad \text { positive integer: xs : positiveInteger } \\
\begin{array}{l}\text { Semantic Meaning: the verbosity level to use where } 1 \text { or less means the bare minimum set of } \\
\text { columns and } 3 \text { or more means the full set of available columns. }\end{array} \\
\begin{array}{ll}\text { Occurrences: } & \text { optional }\end{array}\end{array}$ \\
\hline extras & $\begin{array}{ll}\text { Value type: } & \text { string: xs : string } \\
\text { Semantic Meaning: } & \text { any extra (particularly non-standard) parameters that must be provided } \\
& \text { (apart from what is part of base URL given by the accessURL element). } \\
\text { Occurrences: } & \text { optional } \\
\text { Comments: } & \begin{array}{l}\text { this value should be in the form of name=value pairs delimited with } \\
\text { apersands ( } \&, \text { properly escaped for inclusion into XML). }\end{array}\end{array}$ \\
\hline
\end{tabular}

\subsubsection{SkyPos}

The sia: SkyPos type is used to encode the <testQuery>'s <pos> element, the center position of the test region of interest.

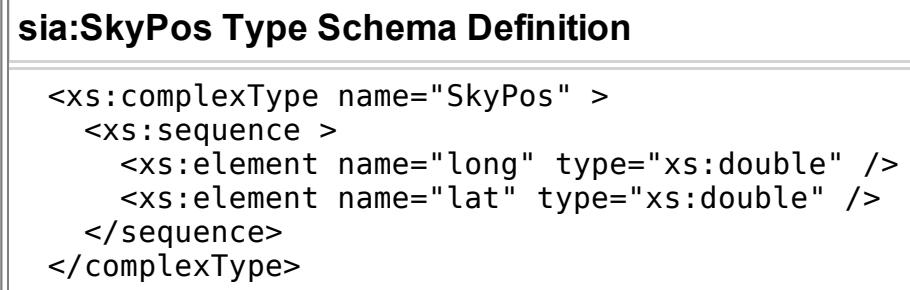

\begin{tabular}{|c|c|c|c|}
\hline \multicolumn{4}{|c|}{ sia:SkyPos Metadata Elements } \\
\hline & & Element & Definition \\
\hline & & long & $\begin{array}{l}\text { Value type: } \quad \text { floating-point number: xs : double } \\
\text { Semantic Meaning: The sky position in the longitude (R.A.) direction } \\
\text { Occurrences: } \quad \text { required }\end{array}$ \\
\hline & & lat & $\begin{array}{l}\text { Value type: } \quad \text { floating-point number: xs : double } \\
\text { Semantic Meaning: The sky position in the latitude (Dec.) direction } \\
\text { Occurrences: } \quad \text { required }\end{array}$ \\
\hline
\end{tabular}




\subsection{Simple Spectral Access}

This section describes the SSA VOResource metadata extension schema which is used to describe services that comply with the Simple Spectral Access protocol. This differs from the other Simple DAL extensions in that it defines two vr: Capability types: ssap: SimpleSpectralAccess and

ssap: ProtoSpectralAccess. The former is intended for services that are intended to be compliant with published SSA Recommendation [SSA]. The latter is intended for services that were deployed prior to the publication of the SSA Recommendation (see section 3.3.3, below).

\subsubsection{The Schema Namespace}

The namespace associated with the SSA extension schema is "http://www.ivoa.net/xml/SSA/v1.1". The namespace prefix, ssap, should be used in applications where common use of prefixes improves interoperability (e.g. in the IVOA registries [RI]). Furthermore, we use the ssap prefix in this document to refer to types defined as part of the SSA extension schema.

Note:

Though it departs a bit from convention, the ssap prefix was chosen to avoid a collision with its use in [SSA] for identifying UTypes from the Spectral Data Model.

\subsubsection{SimpleSpectralAccess}

The ssap: SimpleSpectralAccess type is the vr: Capability sub-type that should be used to describe a service's support for the Simple Spectral Access protocol [SSA]; it is defined as follows:

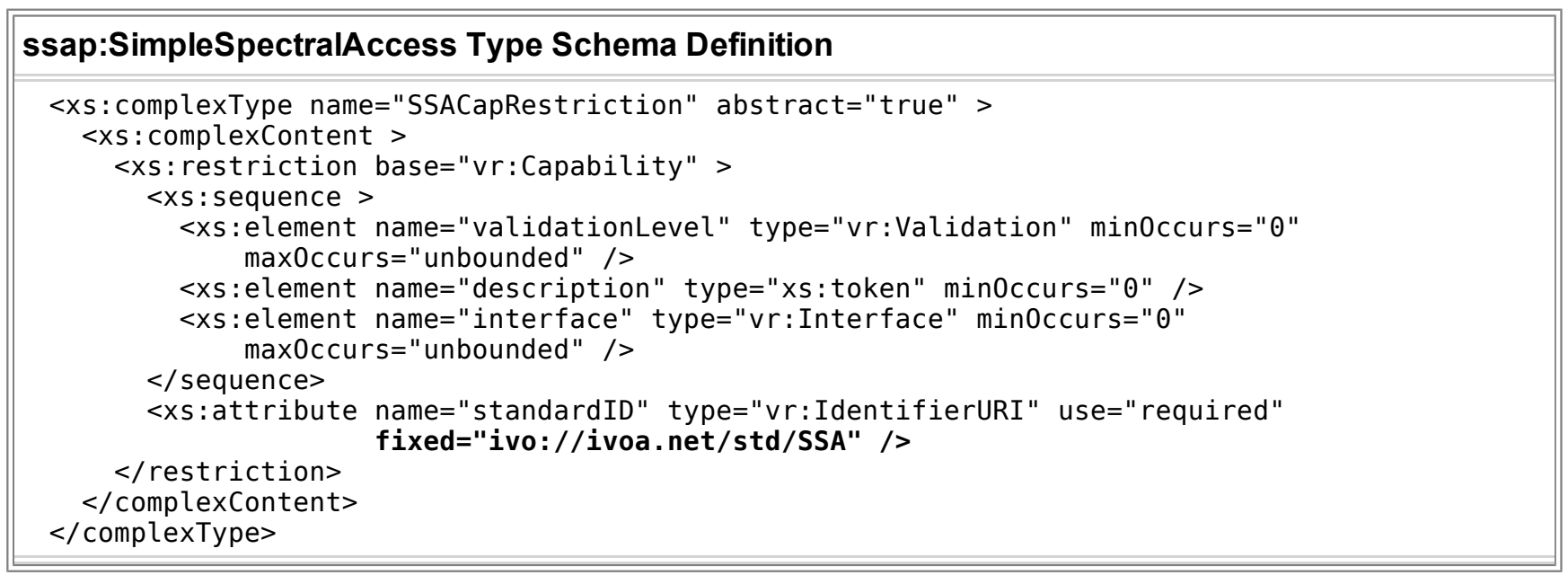

continued on next page 


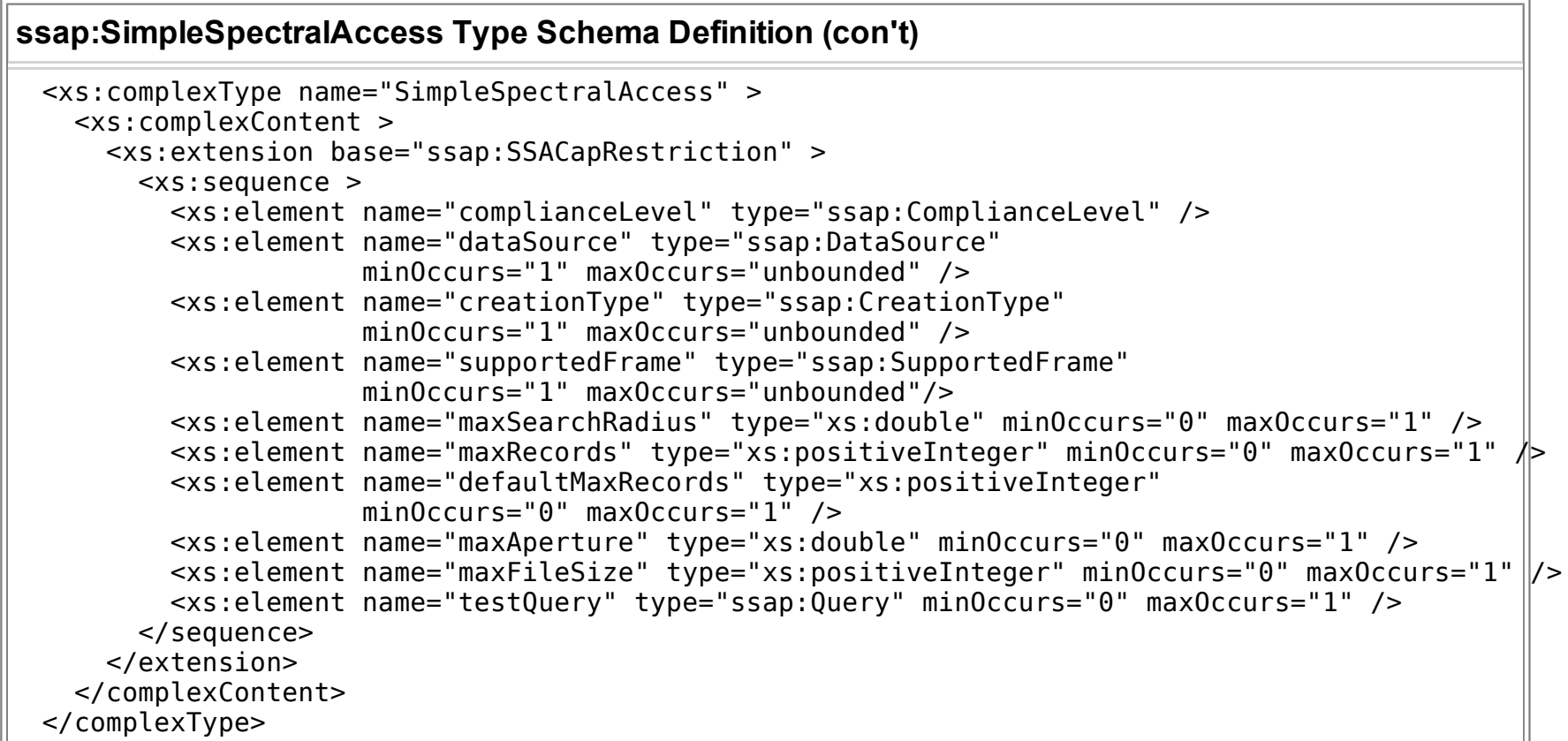

The ssap: SSACapRestriction is defined to fix the value of the standardID attribute; thus, all uses of the ssap: SimpleSpectralAccess type must set standardID to "ivo://ivoa.net/std/SSA". Because the cs: SSACapRestriction is marked as abstract, instance documents can not use it directly as a value for the xsi: type attribute.

The custom metadata that the ssap : SimpleSpectralAccess type provides is given in the table below. Note that some of these elements derive from the SSA standard [SSA]; others, from the RM standard [RM]. The "Semantic Meaning" entry provides the reference to the original definition.

\begin{tabular}{|c|c|c|}
\hline \multicolumn{3}{|c|}{ ssap:SimpleSpectralAccess Extension Metadata Elements } \\
\hline Element & & Definition \\
\hline complianceLevel & 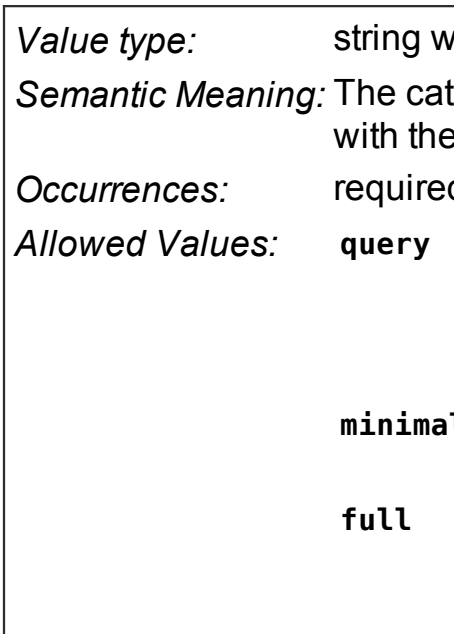 & $\begin{array}{l}\text { th controlled vocabulary } \\
\text { gory indicating the level to which this instance complies } \\
\text { SSA standard, as defined in [SSA], section 1.4.1. } \\
\text { The service supports all of the capabilities and features of } \\
\text { the SSA protocol identified as "must" in the specification, } \\
\text { except that it does not support returning data in at least } \\
\text { one SSA-compliant format. } \\
\text { The service supports all of the capabilities and features of } \\
\text { the SSA protocol identified as "must" in the specification. } \\
\text { The service supports all of the capabilities and features of } \\
\text { the SSA protocol identified as "must" or "should" in the } \\
\text { specification. }\end{array}$ \\
\hline
\end{tabular}




\begin{tabular}{|c|c|c|c|}
\hline \multicolumn{4}{|c|}{ ssap:SimpleSpectralAccess Extension Metadata Elements (con't) } \\
\hline Element & \multicolumn{3}{|r|}{ Definition } \\
\hline \multirow[t]{8}{*}{ dataSource } & \multirow{8}{*}{$\begin{array}{l}\text { Value type: } \\
\text { Semantic Meaning } \\
\text { Occurrences: } \\
\text { Allowed Values: }\end{array}$} & \multicolumn{2}{|c|}{ string with controlled vocabulary } \\
\hline & & \multicolumn{2}{|c|}{$\begin{array}{l}\text { : The category specifying where the data originally came from, as defined } \\
\text { in [SSA], section } 2.5 .1 \text {. }\end{array}$} \\
\hline & & \multicolumn{2}{|c|}{ required; multiple occurrences allowed. } \\
\hline & & survey & $\begin{array}{l}\text { A survey dataset, which typically covers some region of } \\
\text { observational parameter space in a uniform fashion, with } \\
\text { as complete as possible coverage in the region of } \\
\text { parameter space observed. }\end{array}$ \\
\hline & & pointed & $\begin{array}{l}\text { A pointed observation of a particular astronomical object } \\
\text { or field. }\end{array}$ \\
\hline & & custom & $\begin{array}{l}\text { Data which has been custom processed, e.g., as part of a } \\
\text { specific research project. }\end{array}$ \\
\hline & & theory & $\begin{array}{l}\text { Theory data, or any data generated from a theoretical } \\
\text { model, for example a synthetic spectrum. }\end{array}$ \\
\hline & & \multicolumn{2}{|c|}{ artificial Artificial or simulated data. } \\
\hline
\end{tabular}

continued next page 


\begin{tabular}{|c|c|c|c|}
\hline \multicolumn{4}{|c|}{ ssap:SimpleSpectralAccess Extension Metadata Elements (con't) } \\
\hline Element & \multicolumn{3}{|c|}{ Definition } \\
\hline \multirow[t]{11}{*}{ creationType } & \multirow{10}{*}{$\begin{array}{l}\text { Value type: } \\
\text { Semantic Meaning } \\
\text { Occurrences: } \\
\text { Allowed Values: }\end{array}$} & \multicolumn{2}{|c|}{ string with controlled vocabulary } \\
\hline & & \multirow{2}{*}{\multicolumn{2}{|c|}{$\begin{array}{l}\text { The category that describes the process used to produce the dataset, as } \\
\text { defined in [SSA], section } 2.5 .2 \text {. } \\
\text { required; multiple occurrences allowed. }\end{array}$}} \\
\hline & & & \\
\hline & & archival & $\begin{array}{l}\text { The entire archival or project dataset is } \\
\text { returned. Transformations such as metadata or } \\
\text { data model mediation or format conversions } \\
\text { may take place, but the content of the dataset is } \\
\text { not substantially modified (e.g., all the data is } \\
\text { returned and the sample values are not } \\
\text { modified). }\end{array}$ \\
\hline & & cutout & $\begin{array}{l}\text { The dataset is subsetted in some region of } \\
\text { parameter space to produce a subset dataset. } \\
\text { Sample values are not modified, e.g., cutouts } \\
\text { could be recombined to reconstitute the original } \\
\text { dataset. }\end{array}$ \\
\hline & & filtered & $\begin{array}{l}\text { The data is filtered in some fashion to exclude } \\
\text { portions of the dataset, e.g., passing only data } \\
\text { in selected regions along a measurement axis, } \\
\text { or processing the data in a way which } \\
\text { recomputes the sample values, e.g., due to } \\
\text { interpolation or flux transformation. }\end{array}$ \\
\hline & & mosaic & $\begin{array}{l}\text { Data from multiple non- or partially-overlapping } \\
\text { datasets are combined to produce a new } \\
\text { dataset. }\end{array}$ \\
\hline & & projection & $\begin{array}{l}\text { Data is geometrically warped or dimensionally } \\
\text { reduced by projecting through a } \\
\text { multidimensional dataset. }\end{array}$ \\
\hline & & spectralExtraction & $\begin{array}{l}\text { Extraction of a spectrum from another dataset, } \\
\text { e.g., extraction of a spectrum from a spectral } \\
\text { data cube through a simulated aperture. }\end{array}$ \\
\hline & & catalogExtraction & $\begin{array}{l}\text { Extraction of a catalog of some form from } \\
\text { another dataset, e.g., extraction of a source } \\
\text { catalog from an image, or extraction of a line } \\
\text { list catalog from a spectrum (not valid for a SSA } \\
\text { service). }\end{array}$ \\
\hline & Comments: & $\begin{array}{l}\text { Typically this describe } \\
\text { service, but it could de } \\
\text { e.g., if data is partially }\end{array}$ & $\begin{array}{l}\text { s only the processing performed by the data } \\
\text { pribe some additional earlier processing as well, } \\
\text { precomputed. }\end{array}$ \\
\hline
\end{tabular}

continued next page 


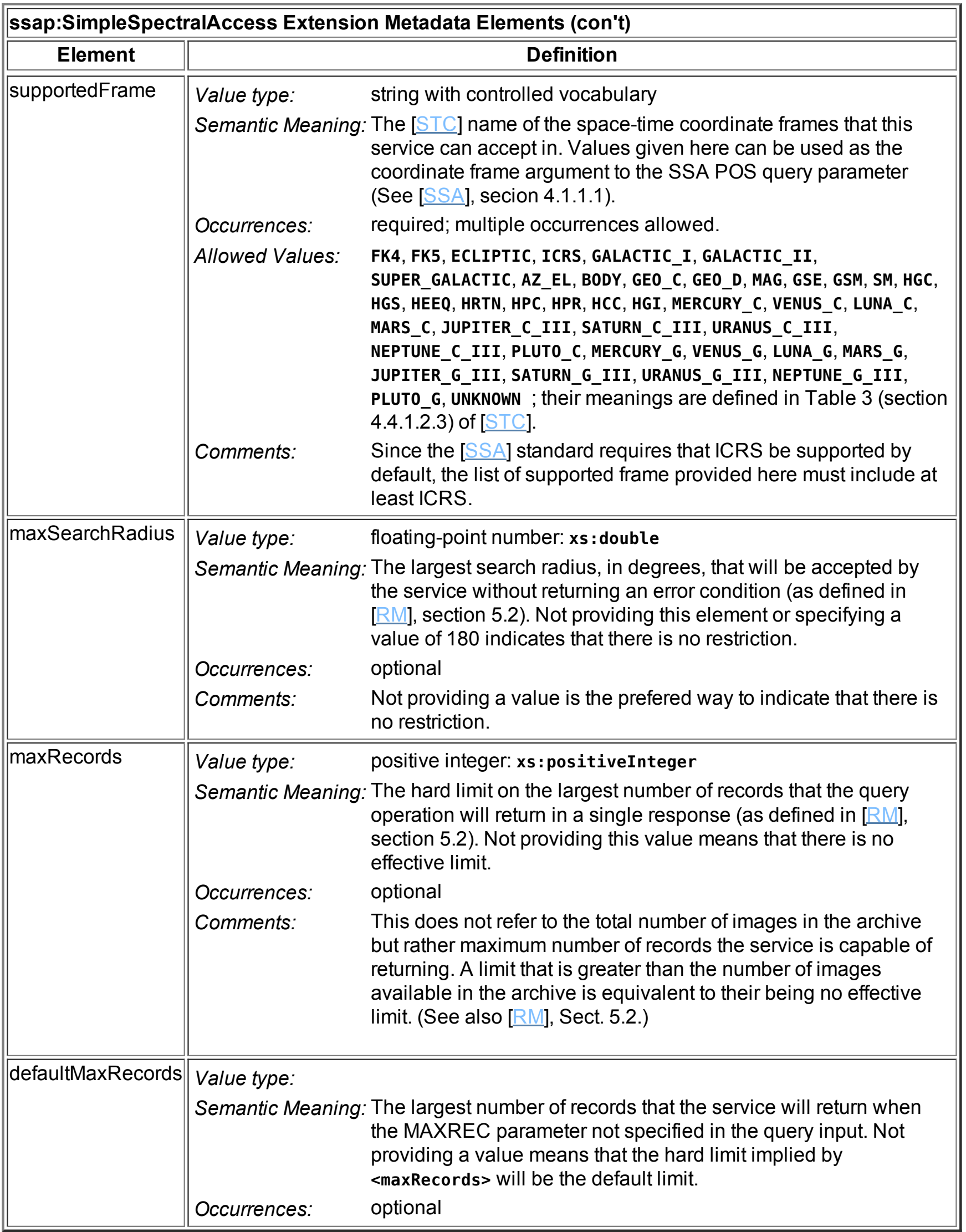

continued next page 


\section{ssap:CreationType Type Schema Definition}

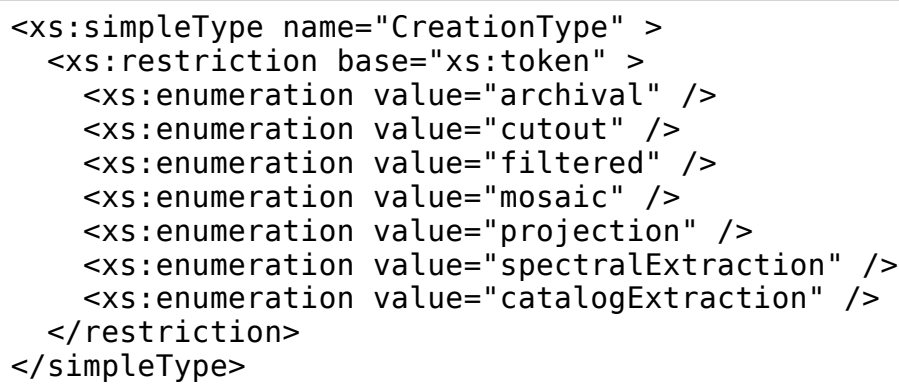

\section{ssap:SupportedFrame Type Schema Definition}

<xs:simpleType name="SupportedFrame" >

$<x s$ : restriction base $=$ "xs: token" >

$<x s$ : enumeration value $=" F K 4 " />$

$<x$ : enumeration value $=" F K 5 " />$

$<x$ : enumeration value $="$ ECLIPTIC" $/>$

$<x$ : enumeration value $=$ "ICRS" />

$<x$ : enumeration value="GALACTIC I" />

$<x$ : enumeration value="GALACTIC II"/>

$<x$ : enumeration value $=$ "SUPER GALACTIC"/>

$<x$ : enumeration value $=" A Z E L " />$

$<x$ : enumeration value $=" B O D Y " />$

$<x$ : enumeration value $=" G E 0 \quad C " />$

$<x$ : enumeration value $=" G E 0$ D" / >

$<x$ : enumeration value $=" M A G " />$

$<x$ : enumeration value $=" \mathrm{GSE} " />$

$<x$ : enumeration value $="$ GSM" $/>$

$<x$ : enumeration value $=" \mathrm{HGC} " />$

$<x$ : enumeration value="HGS" $/>$

$<x$ : enumeration value $=$ "HEEQ" />

$<x$ : enumeration value $=$ "HRTN" />

$<x$ : enumeration value $=" \mathrm{HPC} " />$

$<$ xs: enumeration value $=" H P R " />$

$<x$ : enumeration value $=" \mathrm{HCC} " />$

$<x$ : enumeration value="HGI"/>

$<x$ : enumeration value $=$ "MERCURY C" $/>$

$<x$ : enumeration value $=$ "VENUS $C \bar{C} />$

$<x$ : enumeration value $=$ "LUNA_C" $/>$

$<x$ : enumeration value $=$ "MARS $\mathrm{C}^{-} />$

$<x$ : enumeration value $=$ "JUPITER_C_III"/>

$<x$ : enumeration value $=$ "SATURN $\bar{C} \overline{\text { III" } />}$

$<x$ : enumeration value="URANUS C_III"/>

$<x$ : enumeration value $=$ "NEPTUNE $\bar{C}$ III"/>

$<x$ : enumeration value $=$ "PLUTO $C \bar{C} / \overline{>}$

$<x$ : enumeration value $=$ "MERCURYG" $/>$

$<x$ : enumeration value $=$ "VENUS $G " />$

$<x$ : enumeration value="LUNA $\bar{G} " />$

$<x$ : enumeration value $=$ "MARS $G " />$

$<x$ : enumeration value="JUPITER_G_III"/>

$<$ Xs: enumeration value $=$ "SATURN G III"/>

$<x$ : enumeration value $=$ "URANUS G III" />

$<x$ : enumeration value $=$ "NEPTUNE $\bar{G}$ III"/>

$<x$ : enumeration value $=$ "PLUTO $G " />$

$<x$ : enumeration value $="$ UNKNOWN" $" />$

$<$ /restriction $>$

$</$ simpleType $>$

The definitions of the coordinate reference frames identified in the ssap: SupportedFrame type are the same given in [STC] (and the references therein). 


\subsubsection{1 testQuery and the Query Type}

As with the other DAL vr: capability types, the <testQuery> element is intended to help other VO components (e.g. registries, validation services, services that monitor the VO's operational health--but typically not end users) test that the service is up and operating correctly. It provides a set of legal input parameters that should return a legal response that includes at least matched record. Since this query is intended for testing purposes, the size of the result set should be small.

The ssap : Query type captures the different components of the query into separate elements, as defined below:

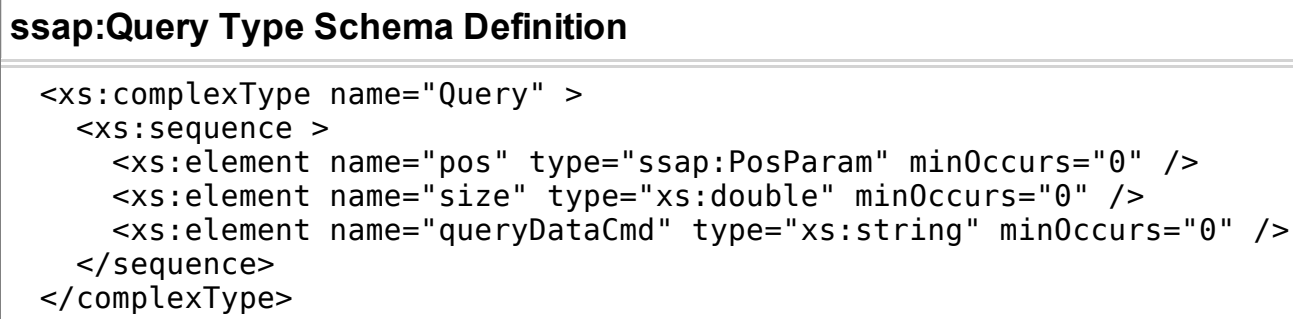

The individual sub-elements are defined as follows:

\begin{tabular}{||l|ll||}
\hline \multicolumn{1}{|c|}{ ssap:Query Metadata Elements } \\
\hline \hline Element & \multicolumn{1}{c|}{ Definition } \\
\hline pos & $\begin{array}{l}\text { Value type: } \\
\text { Semantic Meaning: }\end{array}$ \\
& Occurrences: $\quad$ the center position the search cone given in decimal degrees. \\
& optional
\end{tabular}

\subsubsection{PosParam}

The ssap: PosParam type is used to encode the <testQuery>'s <pos> element, the center position of the test region of interest; it is defined as follows:

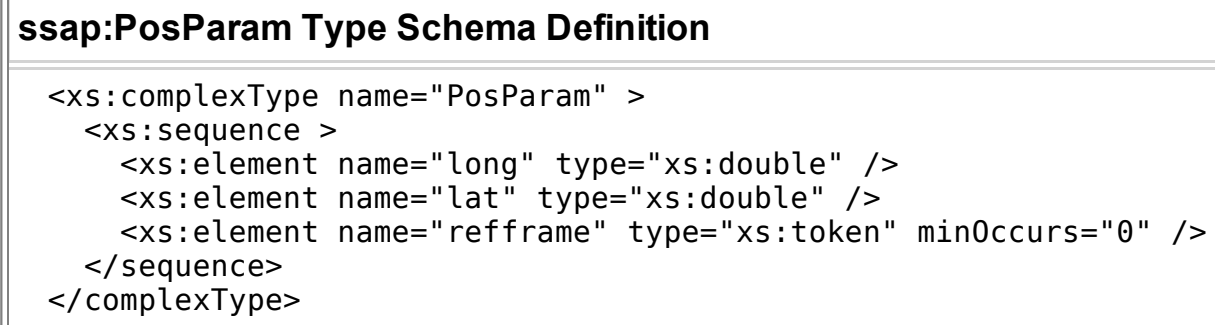


This type differs from the corresponding test position types used by the other DAL extensions in that it allows one to specify a coordinate reference frame supported by SSA's POS input parameter (see [SSA], section 4.1.1.1).

\begin{tabular}{|c|c|}
\hline \multicolumn{2}{|c|}{ ssap:PosParam Metadata Elements } \\
\hline Element & Definition \\
\hline long & 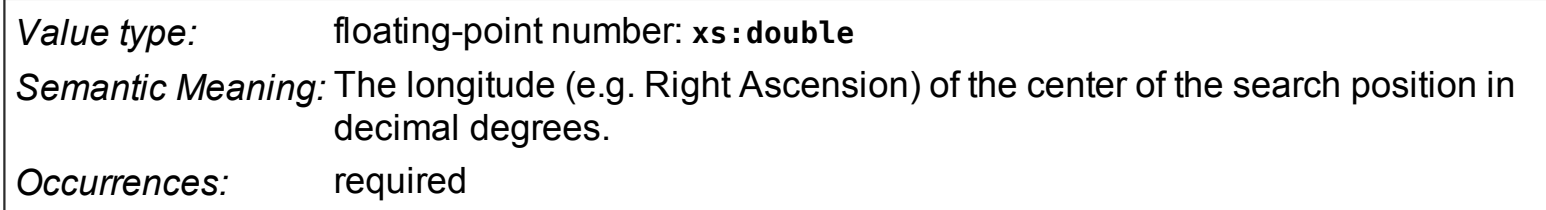 \\
\hline lat & $\begin{array}{ll}\text { Value type: } & \text { floating-point number: xs: double } \\
\text { Semantic Meaning: The latitude (e.g. Declination) of the center of the search position in decimal } \\
\text { degrees. }\end{array}$ \\
\hline refframe & $\begin{array}{l}\text { Value type: } \quad \text { string: } x \text { : token } \\
\begin{array}{ll}\text { Semantic Meaning: the coordinate system reference frame name indicating the frame to assume } \\
\text { for the given position. If not provided, ICRS is assumed. } \\
\text { Occurrences: } \quad \text { optional }\end{array}\end{array}$ \\
\hline
\end{tabular}

\subsubsection{ProtoSpectralAccess}

The ssap: ProtoSpectralAccess type is provided for a special class of SSA services that were historically deployed prior to the publication of the SSA Recommendation [SSA]. An SSA service should describe its support for the protocol using this capability type if it was implemented against an earlier draft of the protocol specification and, therefore, is not expected to be compliant with the actual SSA Recommendation.

This type is defined exactly as the ssap: SimpleSpectralAccess type:

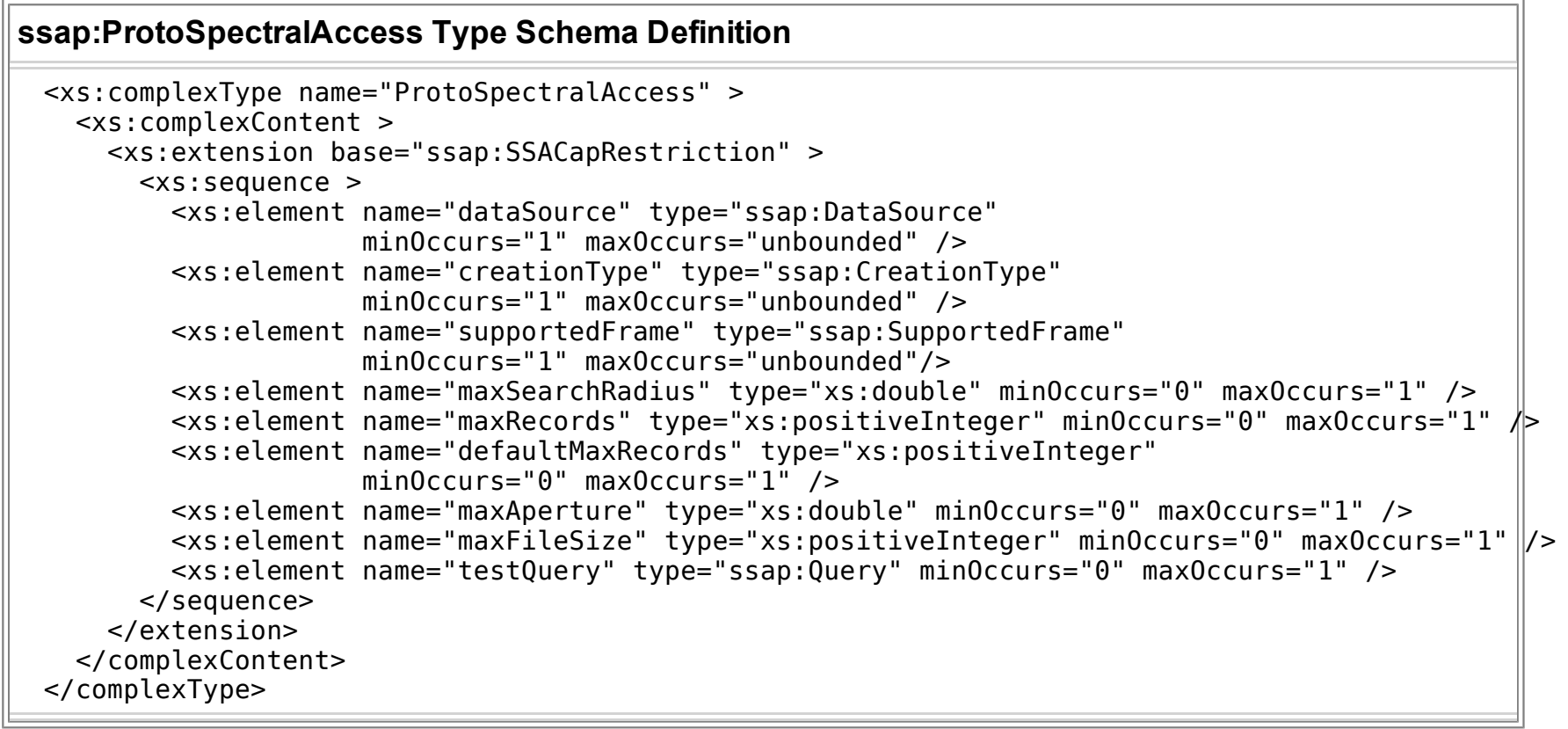

Use of this type is intended to be temporary: providers whose SSA service falls into this category are encouraged to up-date the service for compliance with the final SSA Recommendation. A VOResource resource description must not include both a ssap: SimpleSpectralAccess capability and a ssap: ProtoSpectralAccess capability that describe the same service base URL, as given by the <interface>'s <accessURL>. 


\subsection{Simple Line Access}

This section describes the SLA VOResource metadata extension schema which is used to describe services that comply with the Simple Line Access protocol [SLA].

\subsubsection{The Schema Namespace}

The namespace associated with the SLA extension schema is "http://www.ivoa.net/xml/SLAP/v1.0". The namespace prefix, slap, should be used in applications where common use of prefixes improves interoperability (e.g. in the IVOA registries [RI]). Furthermore, we use the slap prefix in this document to refer to types defined as part of the SLA extension schema.

\subsubsection{SimpleLineAccess}

The slap: SimpleLineAccess type is a vr: Capability sub-type that should be used to describe a service's support for the Simple Line Access protocol [SLA]; it is defined as follows:

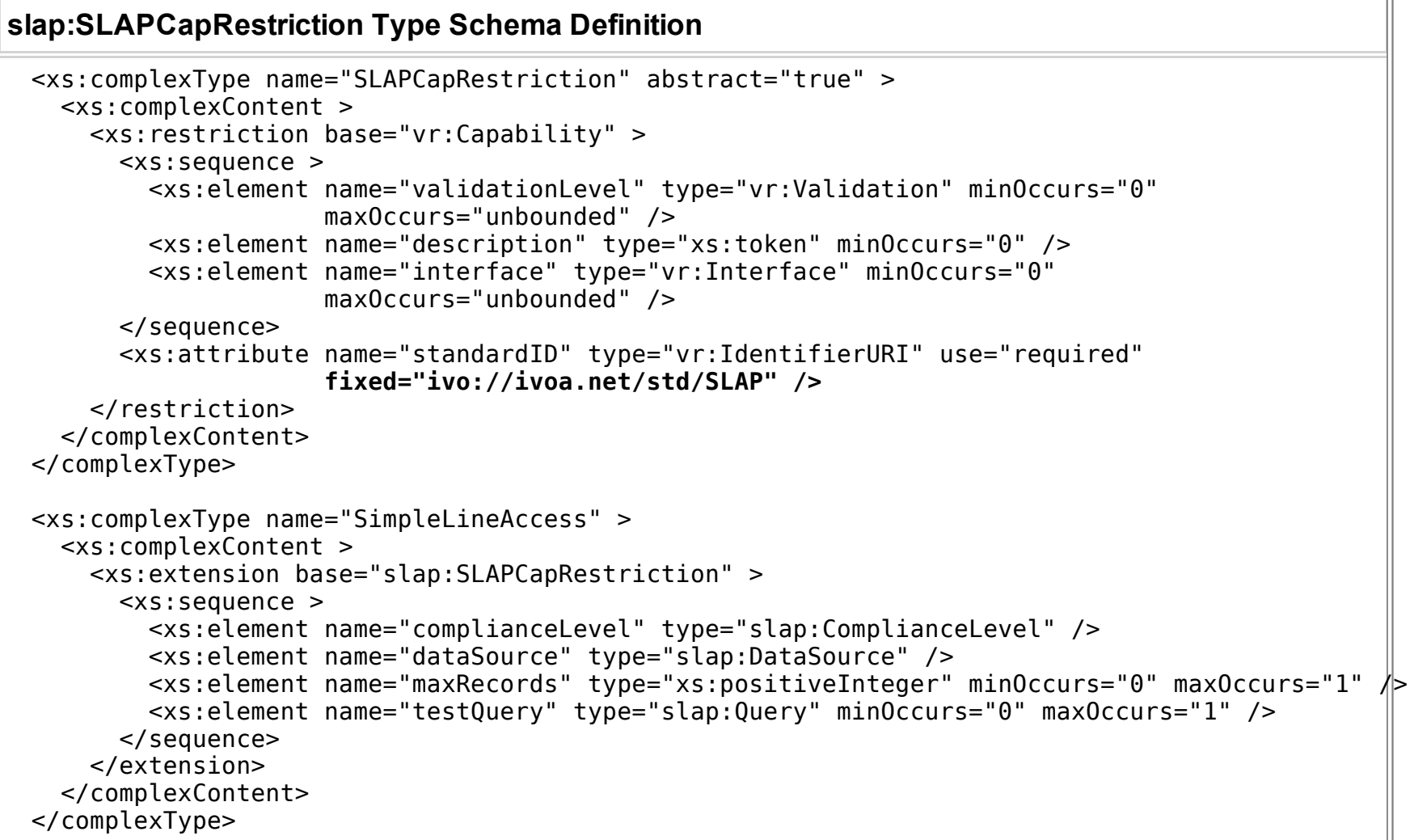


The custom metadata that the slap: SimpleLineAccess type provides is given in the table below.

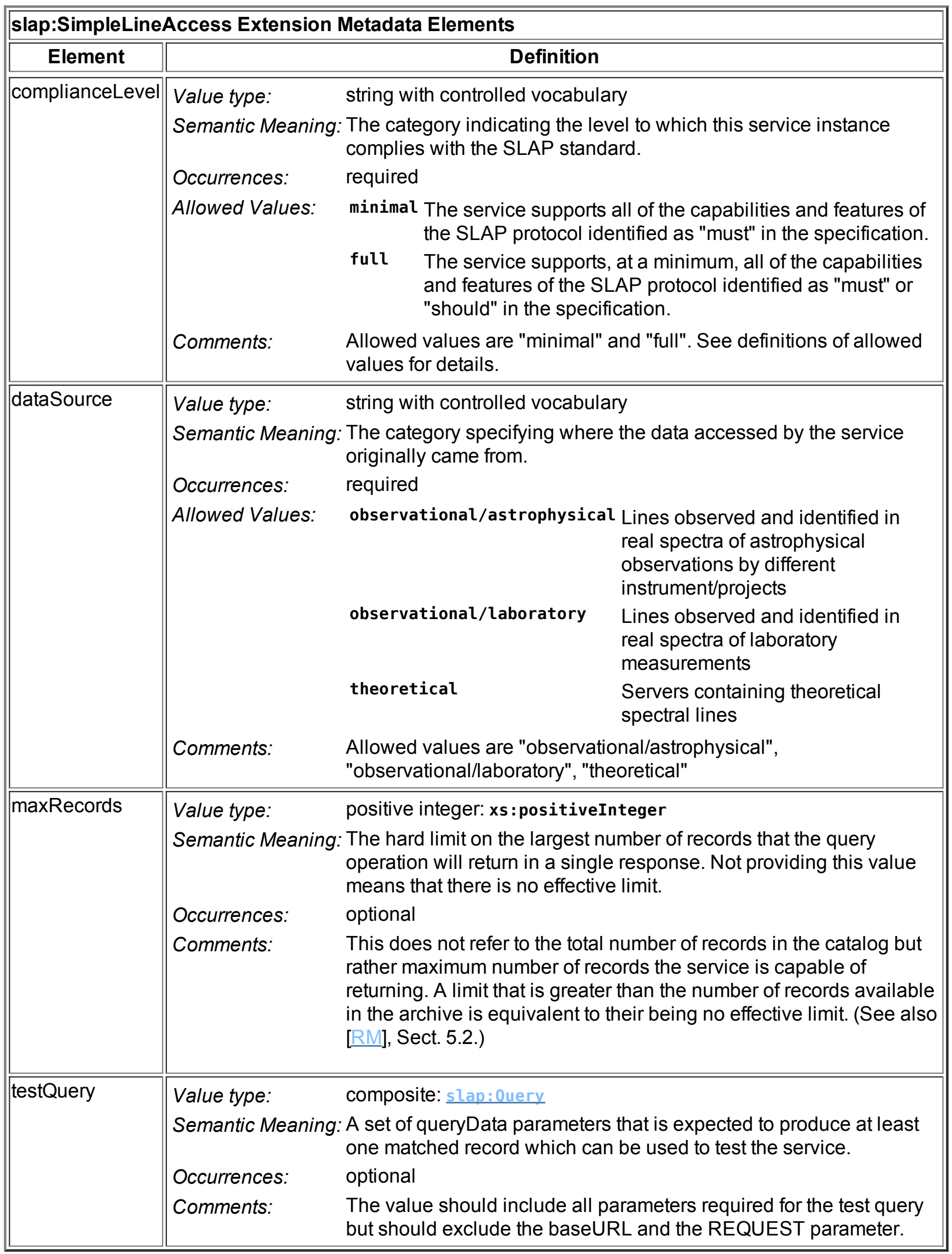


The controlled vocabulary given for the above elements are set by their respective simple types:

\section{slap:ComplianceLevel Type Schema Definition}

<xs:simpleType name="ComplianceLevel" >

$<x$ : restriction base="xs:token" >

$<x$ : enumeration value="minimal" />

$<x$ : enumeration value $="$ full" /

$</$ restriction $>$

$</$ simpleType $>$

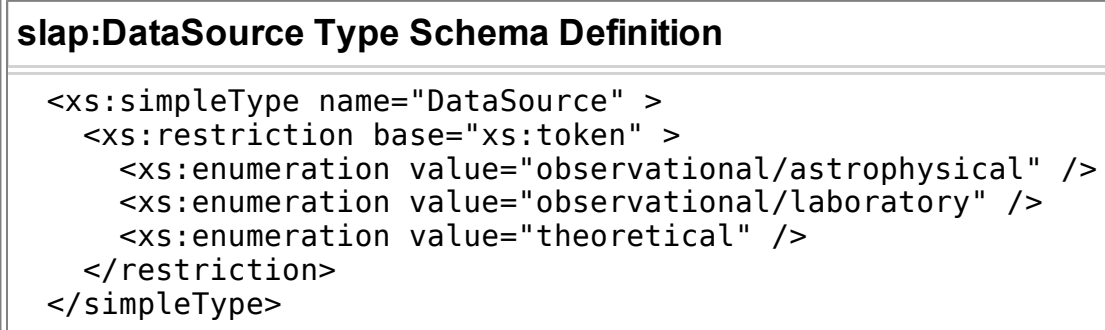

\subsubsection{1 testQuery and the Query Type}

As with the other DAL vr: capability types, the <testQuery> element is intended to help other VO components (e.g. registries, validation services, services that monitor the VO's operational health--but typically not end users) test that the service is up and operating correctly. It provides a set of legal input parameters that should return a legal response that includes at least matched record. Since this query is intended for testing purposes, the size of the result set should be small.

The slap: Query type captures the different components of the query into separate elements, as defined below:

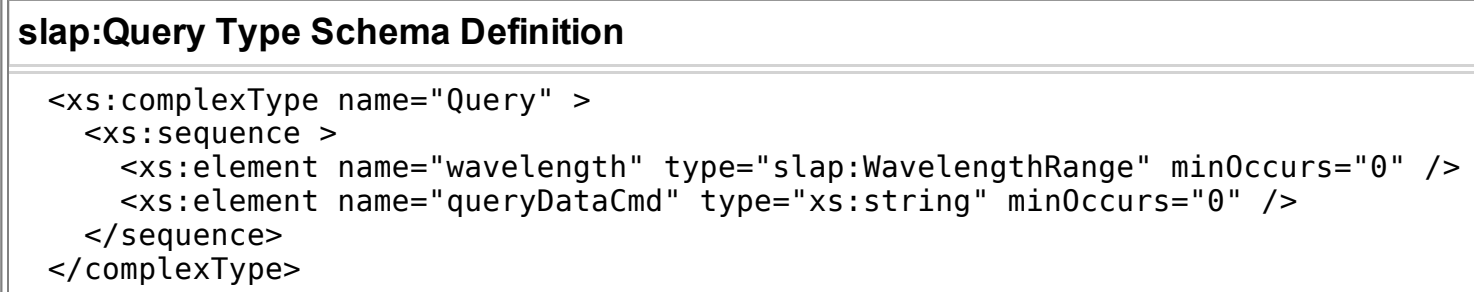


The individual sub-elements are defined as follows:

\begin{tabular}{|c|c|c|}
\hline \multicolumn{3}{|c|}{ slap:Query Metadata Elements } \\
\hline Element & & Definition \\
\hline wavelength & $\begin{array}{l}\text { Value type: } \\
\text { Semantic Meaning: } \\
\text { Occurrences: }\end{array}$ & $\begin{array}{l}\text { Composite: slap: WavelengthRange } \\
\text { Spectral range in meters to be used to constrain the query of spectral } \\
\text { lines. } \\
\text { optional }\end{array}$ \\
\hline queryDataCmd & $\begin{array}{l}\text { Value type: } \\
\text { Semantic Meaning: } \\
\text { Occurrences: } \\
\text { Comments: }\end{array}$ & $\begin{array}{l}\text { string: xs : string } \\
\text { Fully specified queryData test query formatted as an URL argument } \\
\text { list in the syntax specified by the SLAP standard. The list must } \\
\text { exclude the REQUEST argument which is assumed to be set to } \\
\text { "queryData". VERSION may be included if the test query applies to a } \\
\text { specific version of the service protocol. } \\
\text { optional } \\
\text { If queryDataCmd is used to form a query, the default value of } \\
\text { WAVELENGTH specified above is not used; if the test query requires } \\
\text { WAVELENGTH it should be included directly in queryDataCmd. } \\
\text { This value must be a string in the form of name=value pairs delimited } \\
\text { with ampersands (\&). A query may then be formed by appending to } \\
\text { the baseURL the request argument, "REQUEST=queryData\&", } \\
\text { followed by the contents of this element. }\end{array}$ \\
\hline
\end{tabular}

\subsubsection{WavelengthRange}

The slap: WavelengthRange type is used to encode the <testQuery>'s <wavelength> element, the range of wavelengths to search.

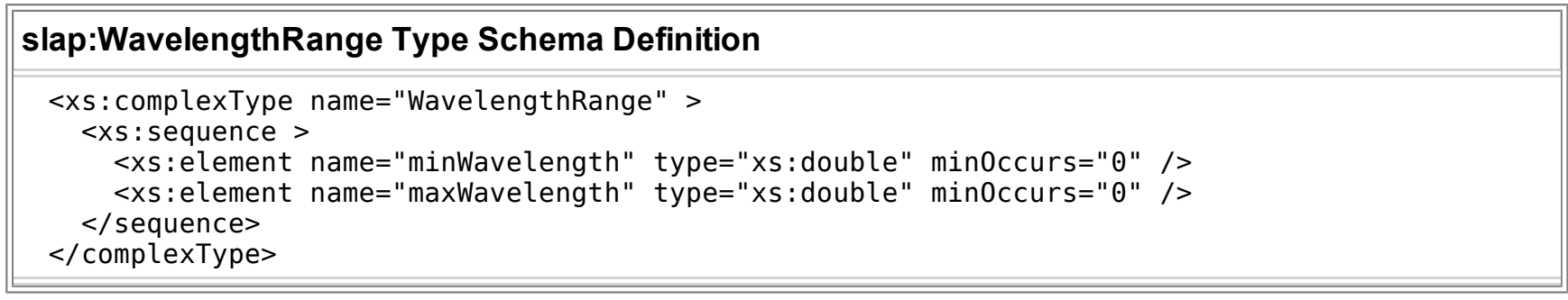

\begin{tabular}{||l|ll||}
\hline \multicolumn{2}{|l||}{ slap:WavelengthRange Metadata Elements } \\
\hline \hline Element & \multicolumn{1}{c|}{ Definition } \\
\hline minWavelength & $\begin{array}{ll}\text { Value type: } \\
\text { Semantic Meaning: Minimum wavelength in meters to be used to constrain the query of } \\
\text { spectral lines } \\
\text { optional }\end{array}$ \\
& Occurrences: $\quad \begin{array}{l}\text { floating-point number: } \mathbf{x s}: \text { double } \\
\text { maxWavelength }\end{array}$ & $\begin{array}{l}\text { Value type: } \\
\text { Semantic Meaning: Maximum wavelength in meters to be used to constrain the query of } \\
\text { spectral lines } \\
\text { optional }\end{array}$ \\
\hline \hline
\end{tabular}




\section{A.1. The ConeSearch XML Schema}

\section{The Complete ConeSearch Schema}

$<$ ?xml version="1.0" encoding="UTF-8"?>

<xs: schema xmlns: xs="http://WwW.w3.org/2001/XMLSchema"

xmlns:vr="http://www. ivoa.net/xml/V0Resource/v1.0"

xmlns: cs="http://www. ivoa. net/xml/ConeSearch/v1.0"

xmlns: vm="http://www. ivoa.net/xml/VoMetadata/v0.1"

targetNamespace $=$ "http: //www. ivoa. net/xml/ConeSearch/v1.0"

elementFormDefault="unqualified" attributeFormDefault="unqualified"

version $=" 1.0 ">$

$<x s$ : annotation>

$<x$ : appinfo>

$<$ vm: schemaName $>$ ConeSea $r$ ch $</ v m$ : schemaName $>$

$<$ vm: schemaPrefix $>$ xs $</$ vm: schemaPrefix $>$

$<$ vm: targetPrefix $>$ cs $</ v m$ : targetPrefix $>$

$</ x s$ :appinfos

$<x s$ : documentation $>$

Implementation of an XML Schema describing a Cone Search Service.

Based on "Simple Cone Search: a First Guide for Data Curators",

http: //www.us-vo.org/metadata/conesearch/.

$</ x s$ : documentation>

$<x s$ : documentation>

This schema defines a Capability type that is specific to Cone

Search. A service that has ConeSearch capability should be

registered as a TabularSkyService. The ConeSearch capability

element must include a ParamHTTP interface.

$</ x s$ : documentation $>$

$</ x s$ : annotation $>$

<xs: import namespace="http://www. ivoa.net/xml/V0Resource/v1.0" schemaLocation="http://www. ivoa.net/xml/VOResource/v1.0"/>

<xs: complexType name="CSCapRestriction" abstract="true">

$<x$ : annotation>

$<x$ : documentation>

an abstract capability that fixes the standardID to the

IVOA ID for the ConeSearch standard.

$</$ xs: documentation $>$

$<x s$ : documentation>

See vr:Capability for documentation on inherited children.

$</ x s$ : documentation $>$

$</ x s$ : annotation $>$

$<x$ : complexContent>

$<x s$ : restriction base="vr:Capability">

$<x s$ : sequence $>$

$<x s$ :element name="validationLevel" type="vr:Validation"

minoccurs $=" \odot$ " max0ccurs $=$ "unbounded" />

$<x s$ : element name="description" type="xs:token" minoccurs $=" \odot " />$

$<x$ : element name="interface" type="vr:Interface" minoccurs $=" 0 "$ max0ccurs $="$ unbounded" $/>$

$</ x$ : sequence $>$

$<x$ :attribute name="standardID" type="vr:IdentifierURI"

$</ x s$ : restriction $>$

use=" required" fixed="ivo://ivoa.net/std/ConeSearch"/>

$</ x s$ : complexContent $>$

$</ x s$ : complexType>

$<x s$ : complexType name="ConeSearch">

$<x s$ : annotation>

$<x s$ : documentation>

The capabilities of a Cone Search implementation.

$</ x s$ : documentation $>$

$</ x s$ : annotation $>$

<xs : complexContent> 


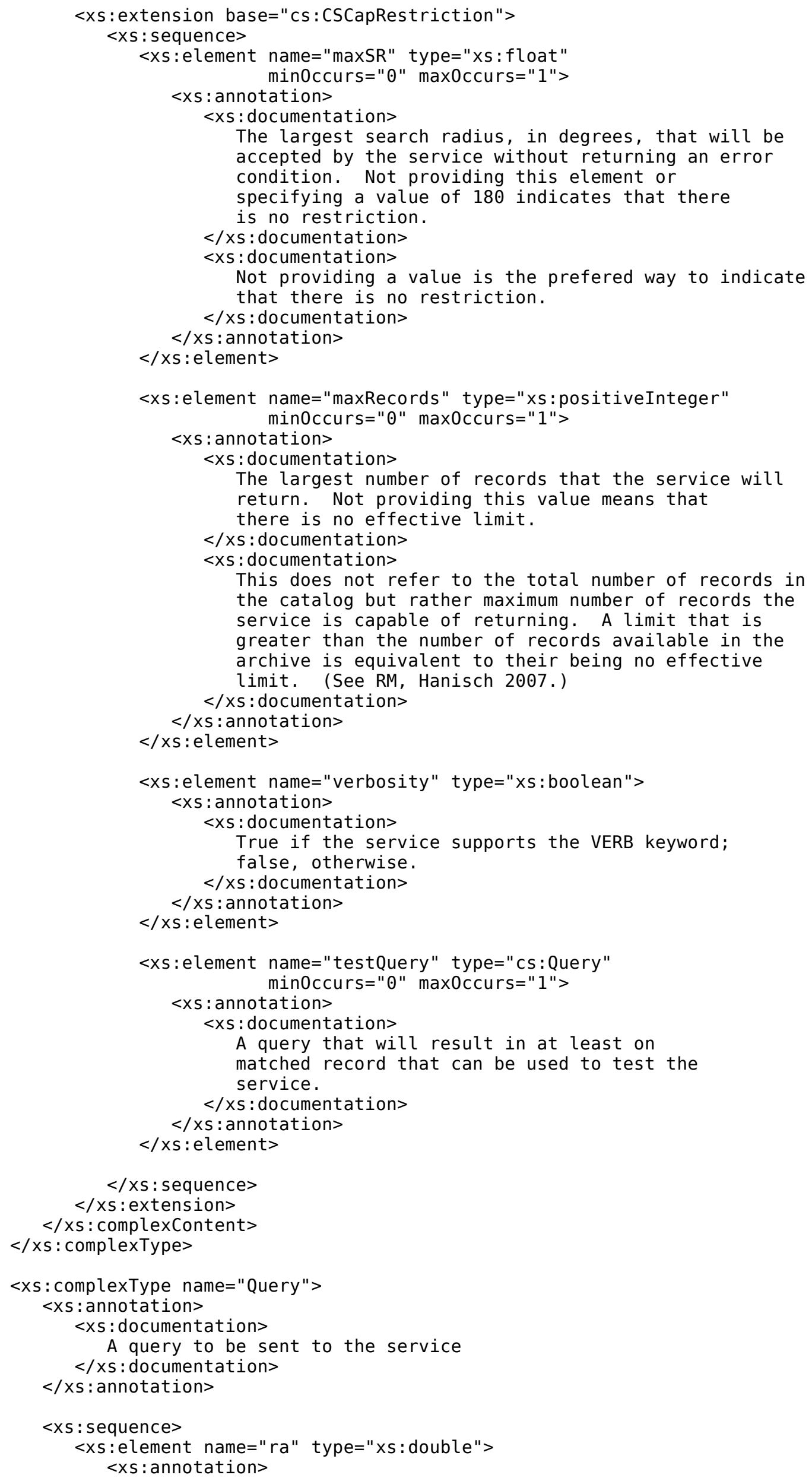




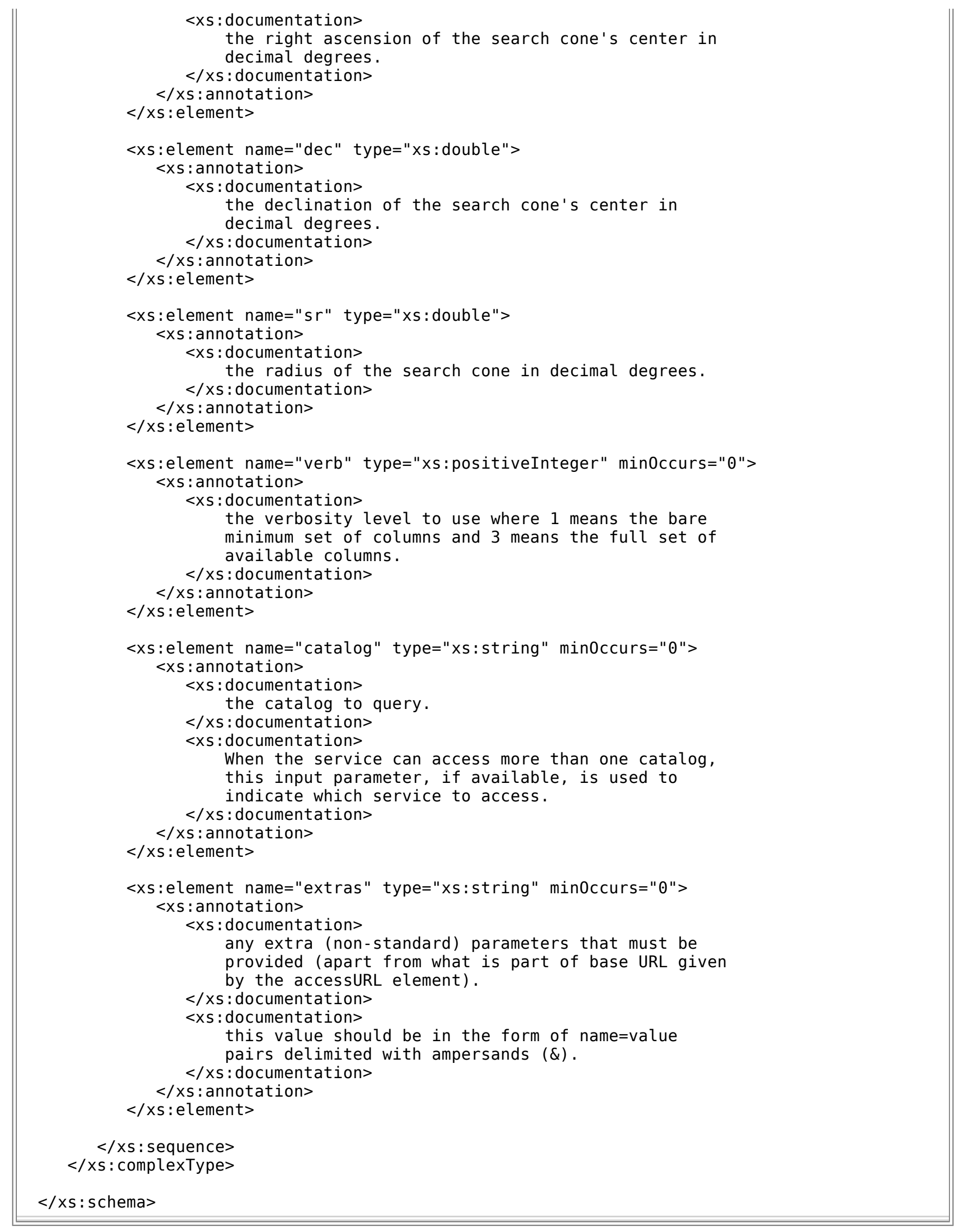




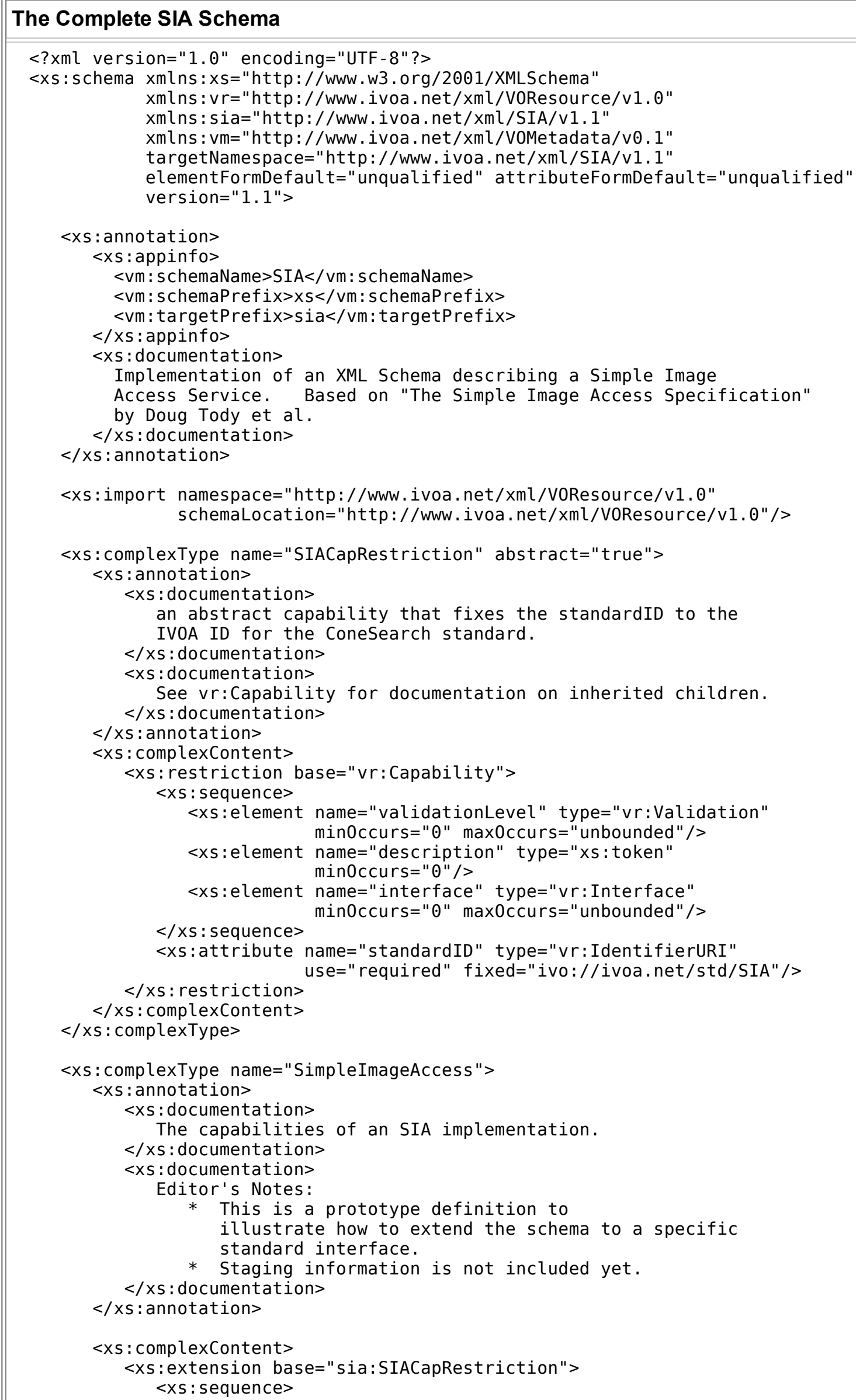




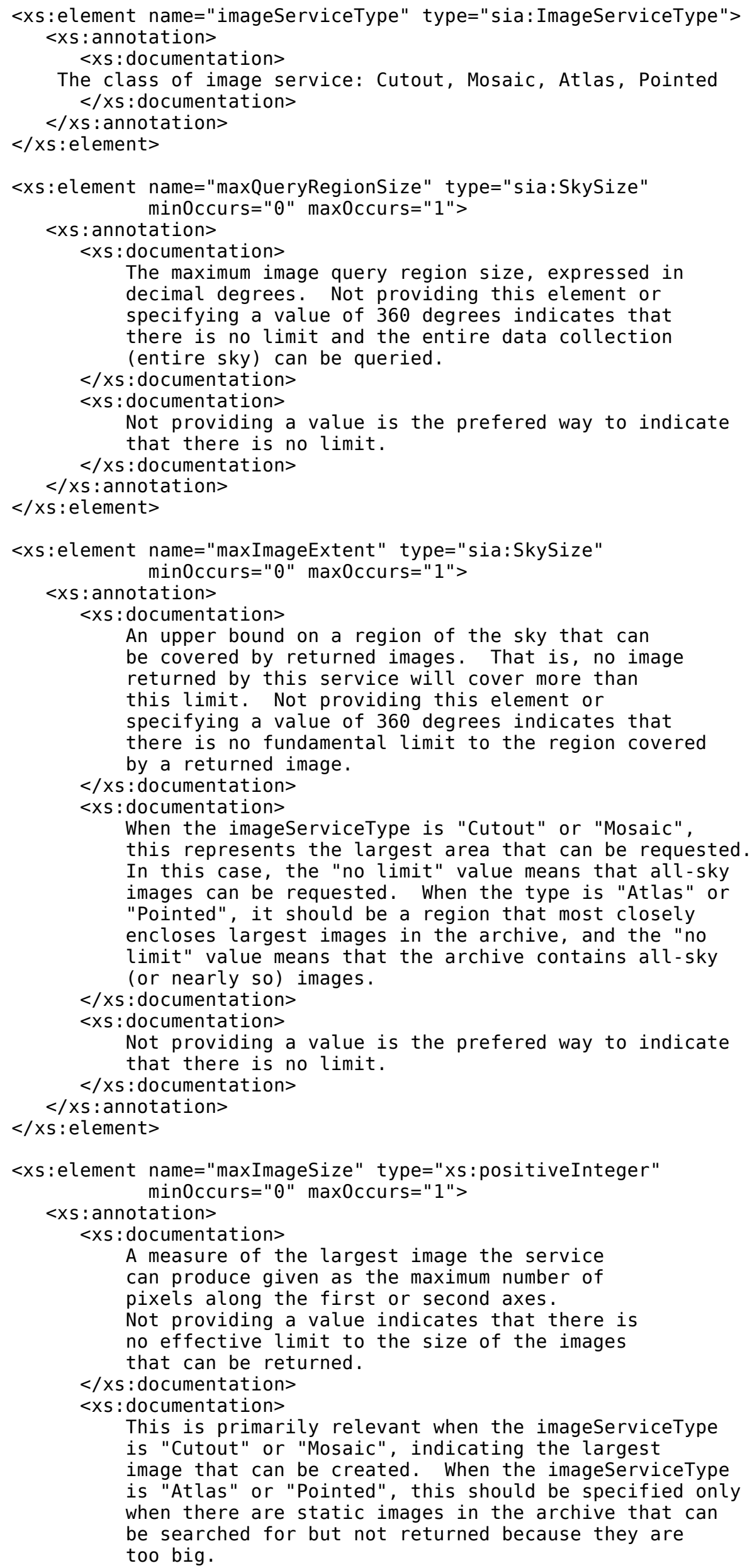




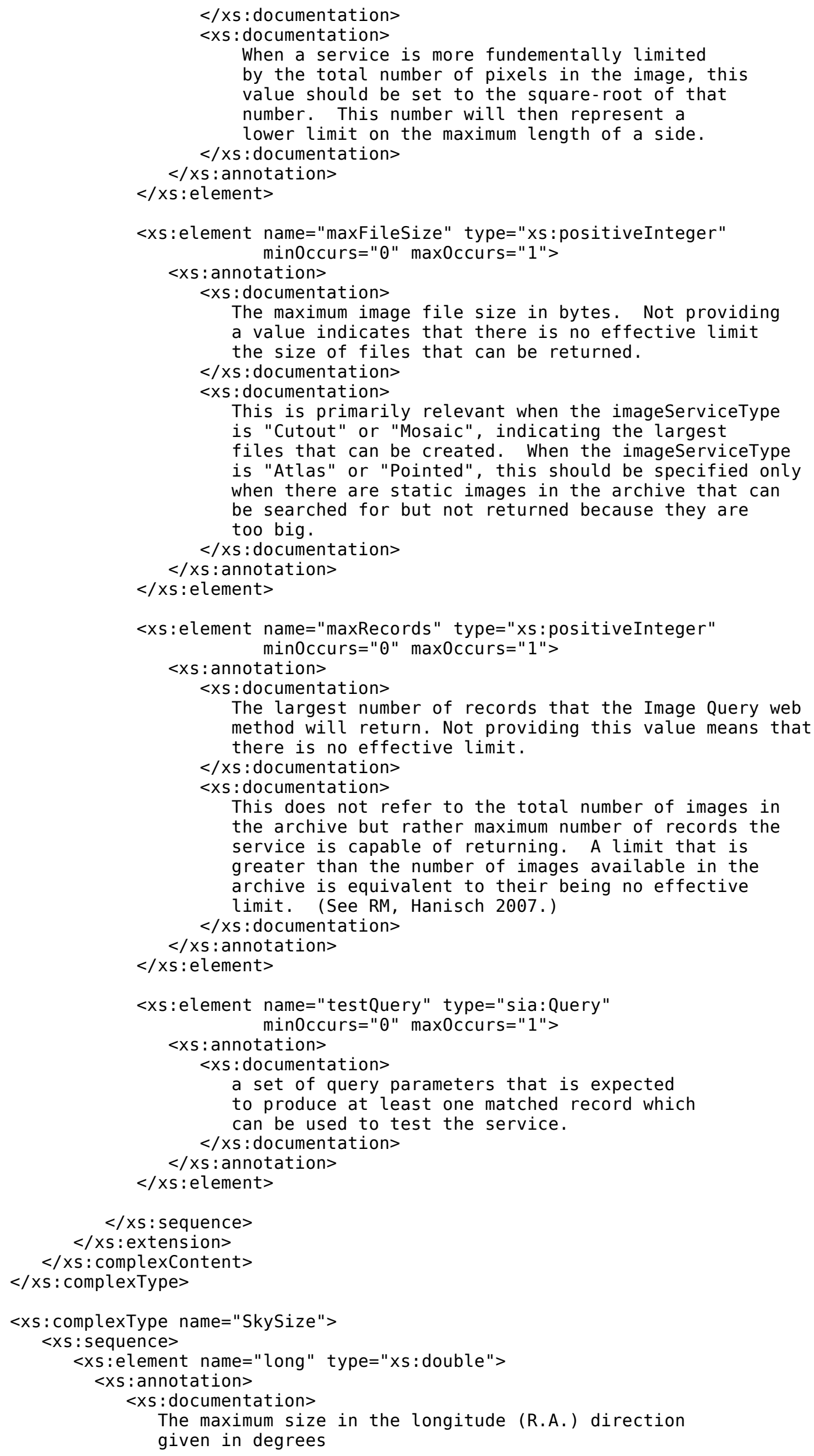




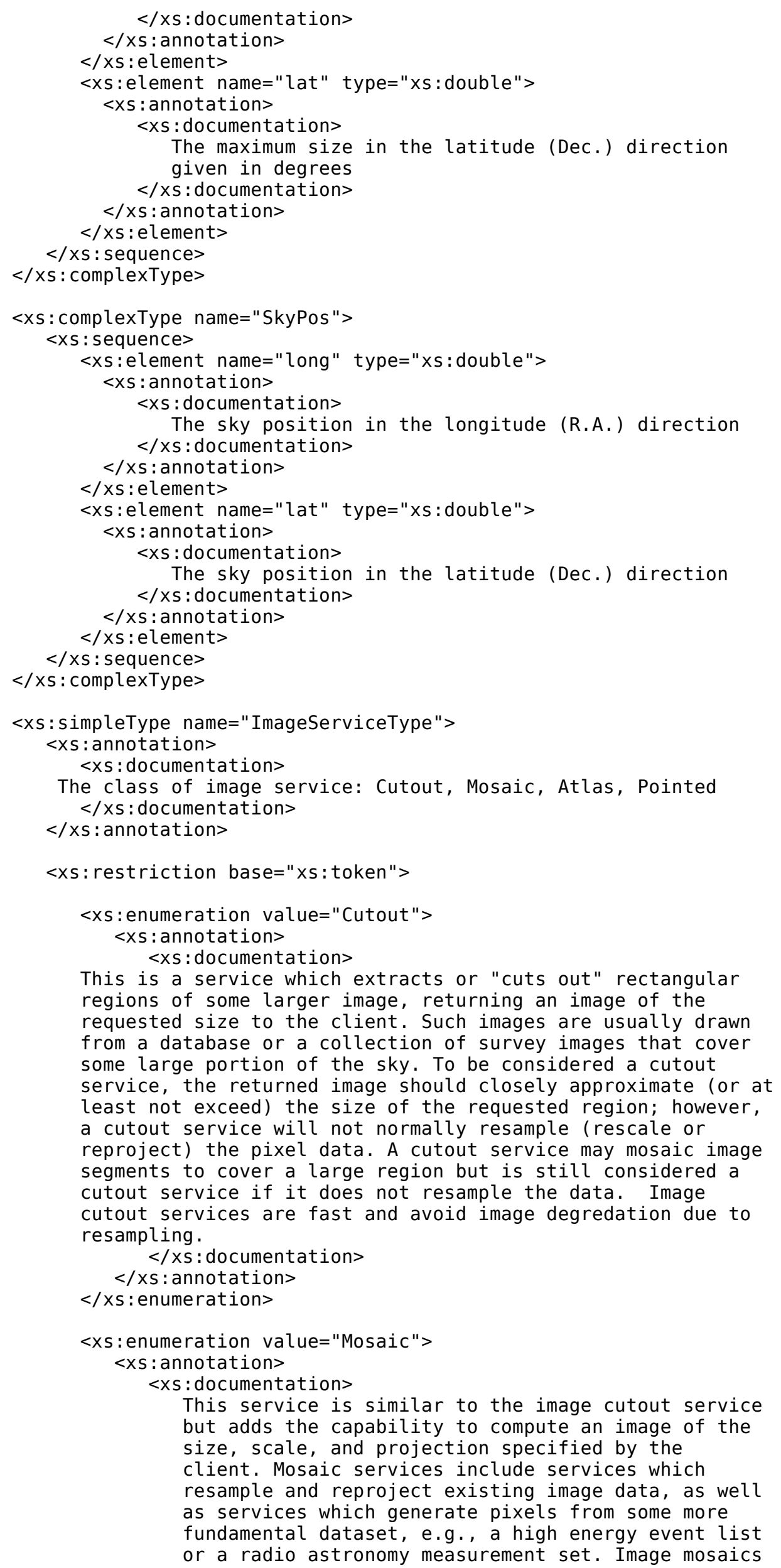


can be expensive to generate for large regions but they make it easier for the client to overlay image data from different sources. Image mosaicing

services which resample already pixelated data will degrade the data slightly, unlike the simpler cutout service which returns the data unchanged.

$</ x s$ : documentation $>$

$</ x s$ : annotation $>$

$</ x s$ : enumeration $>$

$<x$ : enumeration value="Atlas">

$<x$ : annotation>

$<x s$ : documentation>

This category of service provides access to

pre-computed images that make up a survey of some

large portion of the sky. The service, however, is

not capable of dynamically cutting out requested

regions, and the size of atlas images is

predetermined by the survey. Atlas images may range

in size from small cutouts of extended objects to

large calibrated survey data frames.

$</ x s$ : documentation $>$

$</ x s$ : annotation $>$

$</ x s$ : enumeration $>$

$<x$ : enumeration value="Pointed">

$<x$ : annotation>

$<x s$ : documentation>

This category of service provides access to

collections of images of many small, "pointed"

regions of the sky. "Pointed" images normally focus on specific sources in the sky as opposed to being part of a sky survey. This type of service usually applies to instrumental archives from observatories with guest observer programs (e.g., the HST archive) and other general purpose image archives (e.g., the ADIL). If a service provides access to both survey and pointed images, then it should be considered a Pointed Image Archive for the purposes of this specification; if a differentiation between the types of data is desired the pointed and survey data collections should be registered as separate image services.

$</ x s$ : documentation $>$

$</ x s$ : annotation $>$

$</ x s$ : enumeration $>$

$</ x s$ : restriction $>$

$</ x s$ : simpleType>

<xs: complexType name="Query">

$<x$ : annotation>

$<x s$ : documentation>

A query to be sent to the service

$</ x s$ : documentations

$</ x s$ : annotation $>$

$<x s$ : sequence>

$<x s$ :element name="pos" type="sia:SkyPos" min0ccurs $=" \odot ">$

$<x s$ : annotation>

$<x$ : documentation>

the center position of the rectangular region that should be used as part of the query to the SIA service. $</ x s$ : documentation $>$

$</ x s$ : annotation $>$

$</ x s$ : element $>$

$<x s$ :element name="size" type="sia:SkySize" min0ccurs=" $0 ">$ $<x$ : annotation>

$<x$ : documentation>

the rectangular size of the region that should be used as part of the query to the SIA service.

$</$ xs: documentation $>$ 
$</ x s$ : annotation $>$

$</ x s$ : element $>$

$<x s$ :element name="verb" type="xs:positiveInteger" min0ccurs=" $\odot$ ">

$<x$ : annotation>

$<x$ : documentation>

the verbosity level to use where 0 means the bare minimum set of columns and 3 means the full set of available columns.

$</ x s$ : documentation>

$</ x s$ : annotation $>$

$</ x s$ : element $>$

$<x$ : element name="extras" type="xs:string" min0ccurs=" $\theta ">$

$<x$ : annotation>

$<x s$ : documentation>

any extra (particularly non-standard) parameters that must

be provided (apart from what is part of base URL given by the accessURL element).

$</ x s$ : documentation $>$

$<x s$ : documentation>

this value should be in the form of name=value

pairs delimited with apersands $(\&)$.

$</ x s$ : documentation $>$

$</ x s$ : annotation $>$

$</ x s$ : element $>$

$</ x s$ : sequence $>$

$</ x s$ : complexType>

$</$ xs : schema $>$

\section{A.3 The SSA XML Schema}

\section{The Complete SSA Schema}

$<$ ?xml version="1.0" encoding="UTF-8"?>

<xs: schema xmlns: xs="http://www.w3.org/2001/XMLSchema"

xmlns: vr="http://www. ivoa.net/xml/V0Resource/v1.0"

xmlns: ssap="http://www. ivoa. net/xml/SSA/v1.1"

xmlns: vm="http://www. ivoa. net/xml/VoMetadata/v0.1"

targetNamespace $=$ "http: //www. ivoa. net/xml/SSA/v1. 1"

elementFormDefault="unqualified" attributeFormDefault="unqualified"

version="1.1">

$<x$ : annotation>

$<x$ : appinfo>

$<$ vm: schemaName $>$ SSA $</$ vm: schemaName $>$

$<$ vm: schemaPrefix $>$ xs $</$ vm: schemaPrefix $>$

$<$ vm: targetPrefix $>$ ssap</vm: targetPrefix $>$

$</ x$ : appinfo>

$<x s$ : documentation>

XML Schema used to describe the capabilities of a service instance

conforming to the Simple Spectral Access (SSA) protocol.

$</ x s$ : documentation>

$</ x s$ : annotation $>$

<xs: import namespace="http://www. ivoa.net/xml/V0Resource/v1.0" schemaLocation="http://wWw. ivoa.net/xml/V0Resource/v1.0"/>

$<$ ! - - Set the Capability standardID to indicate the SSA protocol. - -

$<x$ : complexType name="SSACapRestriction" abstract="true">

$<x$ : annotation>

<xs: documentation>

An abstract capability that fixes the standardID to the

IVOA ID for the SSA standard.

$</ x S$ : documentation $>$

$<x s$ : documentation>

See vr:Capability for documentation on inherited children.

$</ x s$ : documentation $>$ 


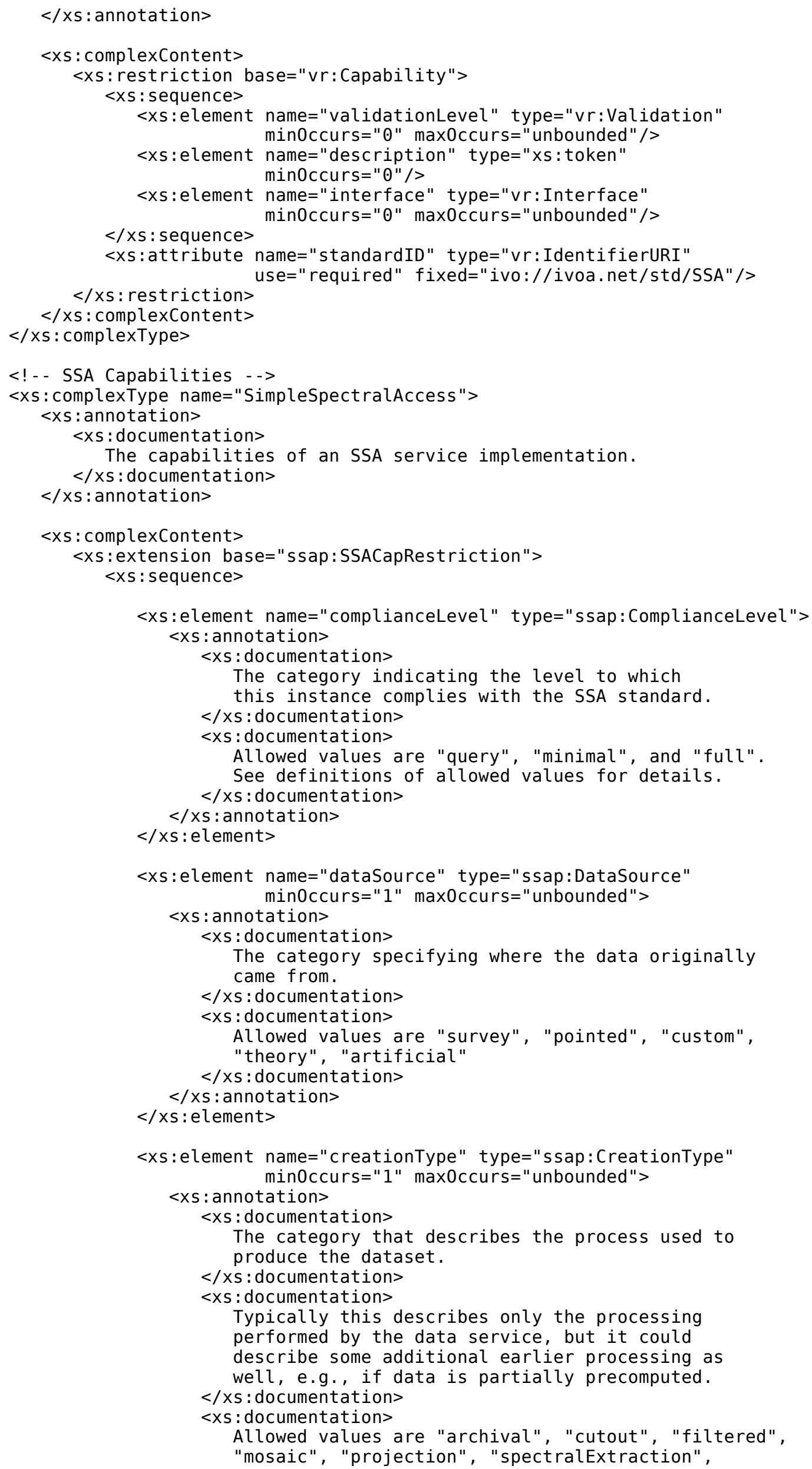




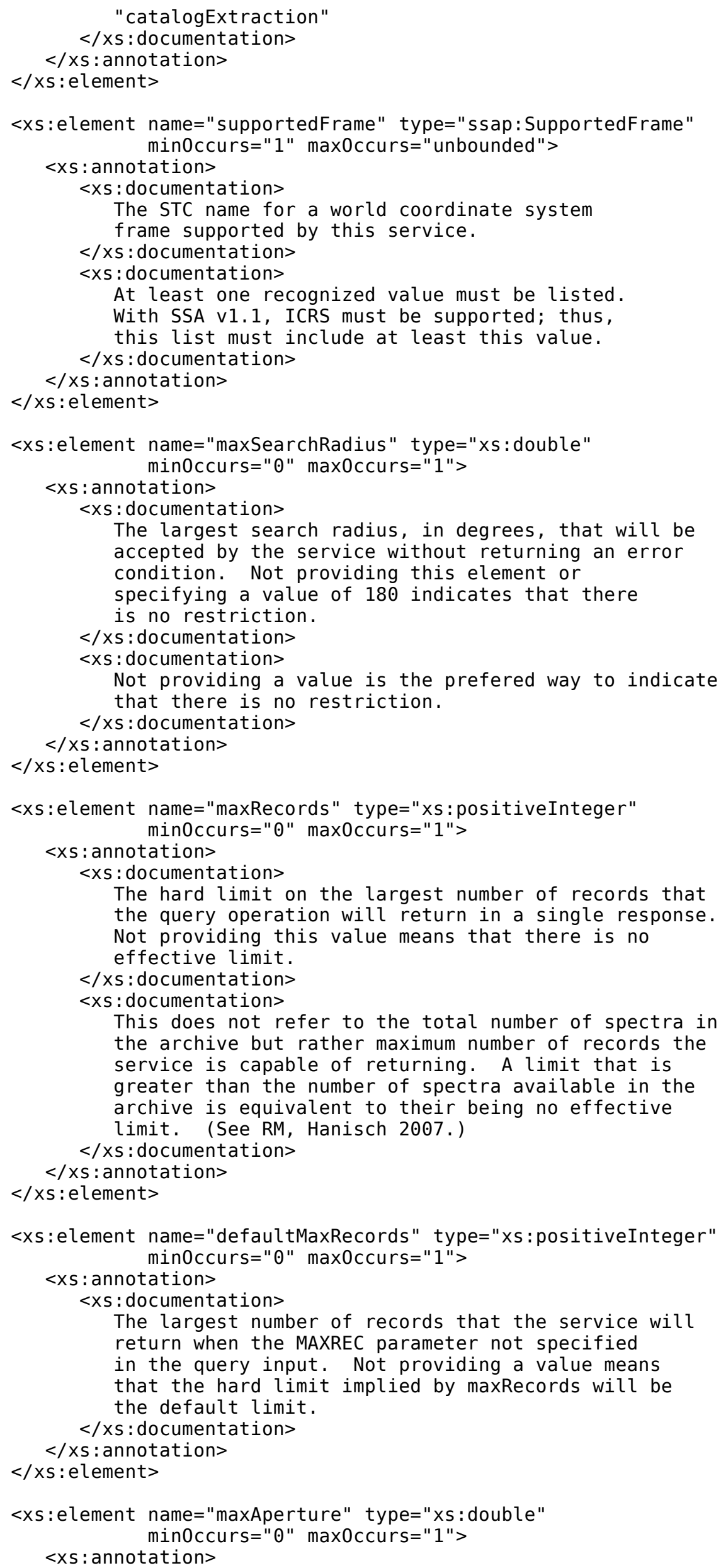


$<x$ : documentation>

The largest aperture that can be supported upon

request via the APERTURE input parameter by a

service that supports the spectral extraction

creation method. A value of 180 or not providing

a value means there is no theoretical limit.

$</ x s$ : documentation $>$

$<x s$ : documentation $>$

Not providing a value is the preferred way to

indicate that there is no limit.

$</ x s$ : documentation $>$

$</ x s$ : annotation $>$

$</ x$ s: element $>$

$<x s$ :element name="maxFileSize" type="xs:positiveInteger"
min0ccurs=" $\bullet "$ maxoccurs="1">

$<x$ : annotation $>$

$<x s$ : documentation>

The maximum spectrum file size in bytes that will

be returned. Not providing

a value indicates that there is no effective limit the size of files that can be returned.

$</ x s$ : documentation $>$

$<x$ : documentation $>$

This is primarily relevant when spectra are created on the fly (see creationType). If the service provides access to static spectra, this should only be specified if there are spectra in the archive that can be searched for but not returned because they are too big.

$</ x s$ : documentation $>$

$</ x s$ : annotation $>$

$</ x s$ : element $>$

<xs:element name="testQuery" type="ssap:Query" min0ccurs $=" \Theta "$ max0ccurs $=" 1 ">$

$<x$ : annotation>

$<x s$ : documentation>

a set of query parameters that is expected to produce at least one matched record which can be used to test the service.

$</ x s$ : documentation $>$

$</ x$ : annotation $>$

$</ x s$ : element $>$

$</ x s$ : sequence $>$

$</ x$ : extension>

$</ x s$ : complexContent $>$

$</ x s$ : complexType>

$<x s:$ simpleType name="ComplianceLevel ">

$<x$ : annotation>

$<x s$ : documentation>

The allowed values for indicating the level at which a

service instance complies with the SSA standard.

$</ x s$ : documentation $>$

$</ x s$ : annotation $>$

$<x s$ : restriction base $=" x s:$ token" $>$

$<x$ : enumeration value $=$ "query" >

$<x$ : annotation>

$<x$ : documentation>

The service supports all of the capabilities and features

of the SSA protocol identified as "must" in the

specification, except that it does not support returning data in at least one SSA-compliant format.

$</ x s$ : documentation $>$

$<x s$ : documentation $>$

This level represents the lowest level of compliance.

$</ x s$ : documentations

$</ x$ : annotation $>$

$</ x s$ : enumeration $>$ 


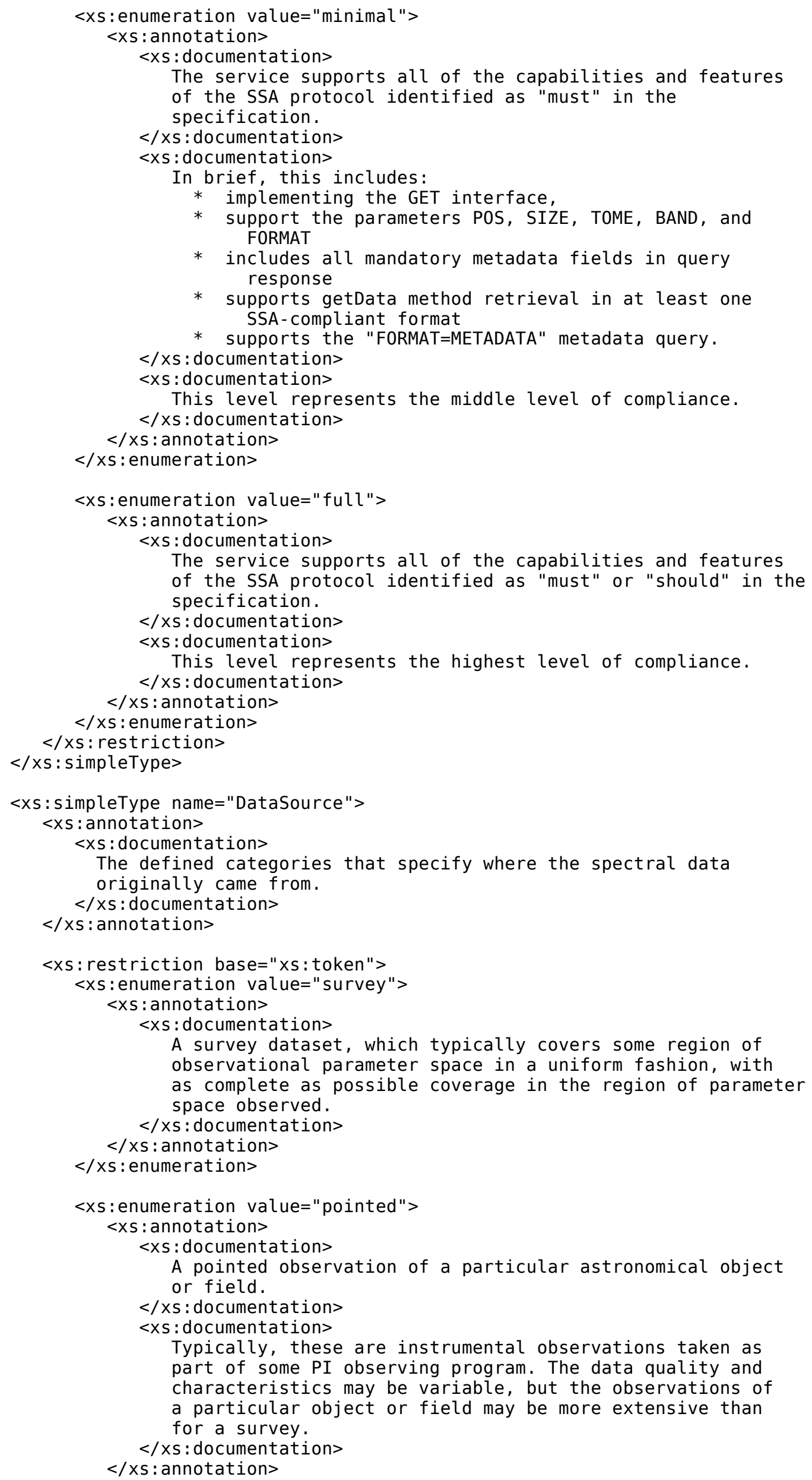




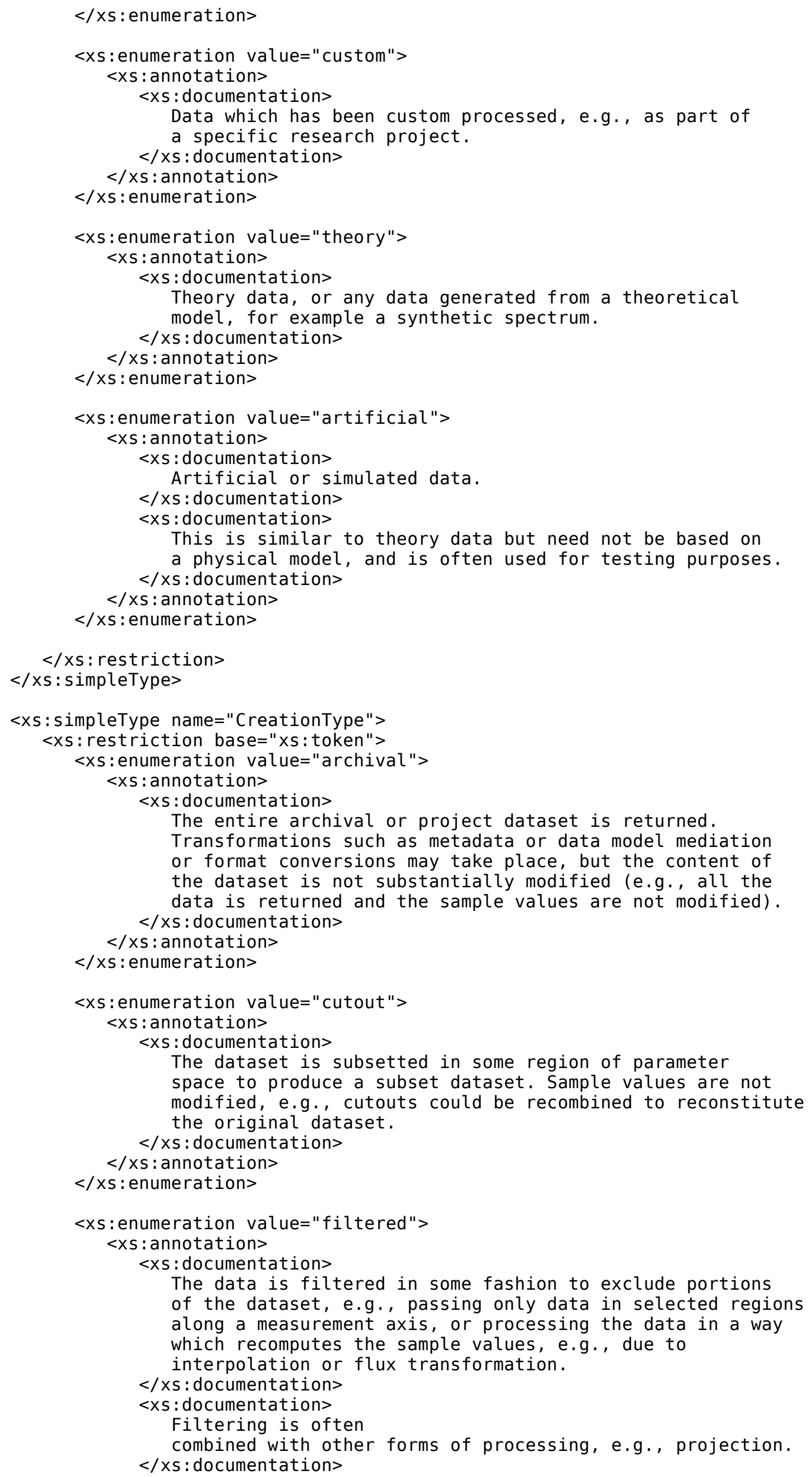




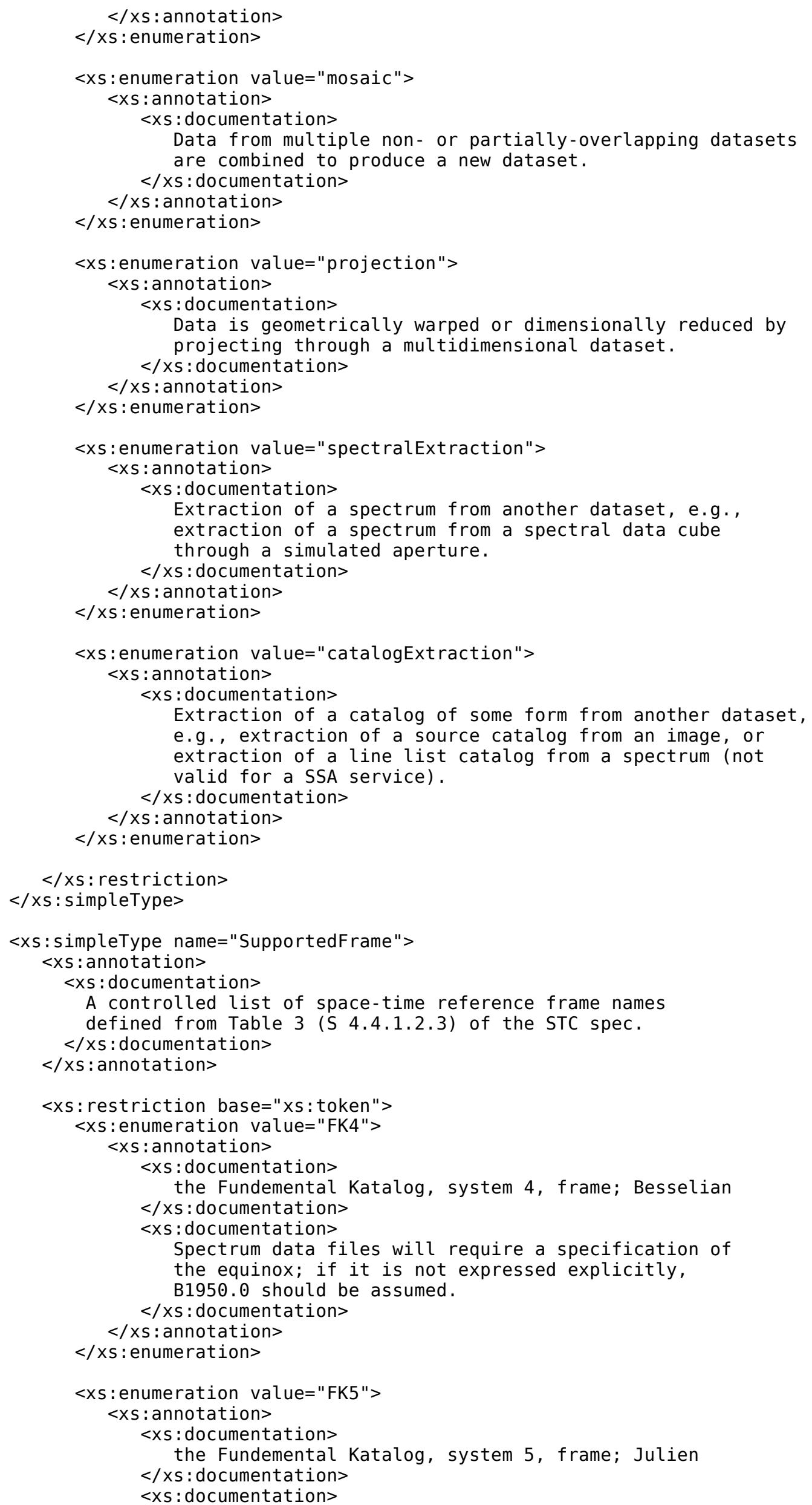


Spectrum data files will require a specification of the equinox; if it is not expressed explicitly, J2000.0 should be assumed.

$</ x s$ : documentation $>$

$</ x s$ : annotation $>$

$</ x s$ : enumeration $>$

$<x$ : enumeration value="ECLIPTIC" >

$<x$ : annotation>

$<x s$ : documentation>

Ecliptic coordinates

$</ x s$ : documentation $>$

$</ x s$ : annotation $>$

$</ x s$ : enumeration $>$

$<x$ : enumeration value $=$ "ICRS" $>$

$<x$ : annotation>

<xs: documentation>

International Celestial Reference System

$</ x s$ : documentation $>$

$</ x s$ : annotation $>$

$</ x s$ : enumeration $>$

<xs: enumeration value="GALACTIC_I">

$<x s$ : annotation>

<xs: documentation>

old Galactic coordinates

$</ x s$ : documentation $>$

$</ x s$ : annotation $>$

$</ x s$ : enumeration $>$

$<$ Xs: enumeration value="GALACTIC II">

$<x$ : annotation>

$<x$ : documentation>

old Galactic coordinates

$</ x s$ : documentation $>$

$</ x s$ : annotation $>$

$</ x s$ : enumeration $>$

$<x$ : enumeration value="SUPER_GALACTIC" >

$<x$ : annotation>

$<x s$ : documentation>

Super-galactic coordinates with the north pole at

GALACTIC_II $(47.37,+6.32)$ and the origin at

GALACTIC II $(137.37,0)$.

$</ x s$ : documentations

$</ x s$ : annotation $>$

$</ x s$ : enumeration $>$

$<x$ : enumeration value $=" A Z$ EL">

$<x$ : annotation>

$<x$ : documentation>

The local azimuth and elevation frame where azimuth

increases from north through east.

$</ x s$ : documentation $>$

$</ x s$ : annotation $>$

$</ x s$ : enumeration $>$

$<x$ : enumeration value="BODY">

$<x$ : annotation>

<xs: documentation>

A generic solar system body-centered coordinate frame

$</ x s$ : documentation $>$

$<x$ : documentation $>$

If applicable, queries against this system should assume a default magnitude value or range in the absence of an applicable (non-standard) query constraint. Service providers are encouraged to document the such assumptions in the resource or capability description.

$</ x s$ : documentations

$</ x$ : annotation $>$

$</ x s$ : enumeration $>$ 


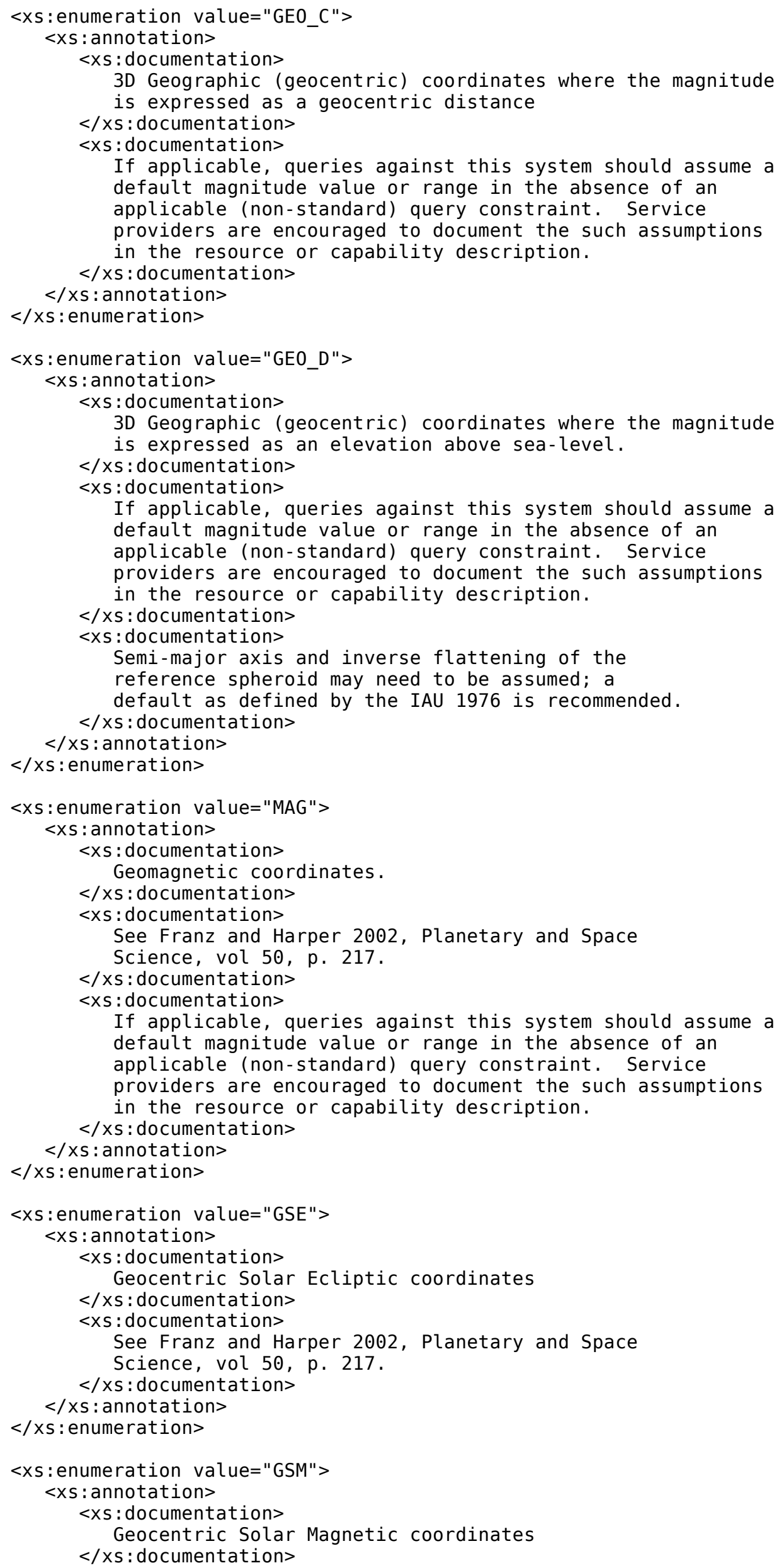


$<x$ : documentation>

See Franz and Harper 2002, Planetary and Space

Science, vol 50, p. 217.

$</ x s$ : documentation $>$

$</ x s$ : annotation $>$

$</ x s$ : enumeration $>$

$<x$ : enumeration value $=" H G C ">$

$<x$ : annotation>

$<x$ : documentation>

Heliographic coordinates (Carrington)

$</ x s$ : documentation $>$

$<x$ : documentation $>$

See Thompson 2006, "Coordinate Systems for Solar

Image Data", A\&A., Section 2.2

$</ x s$ : documentation $>$

$</ x s$ : annotation $>$

$</ x s$ : enumeration $>$

$<x$ : enumeration value="HGS">

$<x$ : annotation>

$<x$ : documentation>

Heliographic coordinates (Stonyhurst)

$</ x s$ : documentation $>$

$<x s$ : documentation>

See Thompson 2006, "Coordinate Systems for Solar

Image Data", A\&A., Section 2.2

$</ x s$ : documentation $>$

$</ x s$ : annotation $>$

$</ x s$ : enumeration $>$

$<x$ : enumeration value="HEEQ" >

$<x$ : annotation>

$<x$ : documentation>

Heliographic Earth Equatorial coordinates

$</ x s$ : documentation $>$

$<x s$ : documentation>

See Franz and Harper 2002, Planetary and Space

Science, vol 50, p. 217, and

Thompson 2006, "Coordinate Systems for Solar

Image Data", A\&A., Section 2.1

$</ x s$ : documentation $>$

$</ x$ : annotation $>$

$</ x s$ : enumeration $>$

$<x$ : enumeration value $="$ HRTN">

$<x$ : annotation>

$<x s$ : documentation>

Heliographic Radial-Tangential-Normal coordinates

$</ x s$ : documentation $>$

$<x$ : documentation $>$

See Franz and Harper 2002, Planetary and Space

Science, vol 50, p. 217.

$</ x s$ : documentation $>$

$</ x s$ : annotation $>$

$</ x s$ : enumeration $>$

$<x$ : enumeration value="HPC" >

$<x$ : annotation>

$<x$ : documentation $>$

Helioprojective Cartesian coordinates

$</ x s$ : documentation $>$

$<x s$ : documentation>

See Thompson 2006, "Coordinate Systems for Solar

Image Data", A\&A., Section 2.1

$</ x s$ : documentation $>$

$</ x s$ : annotation $>$

$</ x s$ : enumeration $>$

<xs: enumeration value="HPR">

$<x$ : annotation>

$<x s$ : documentation>

Helioprojective Polar coordinates 


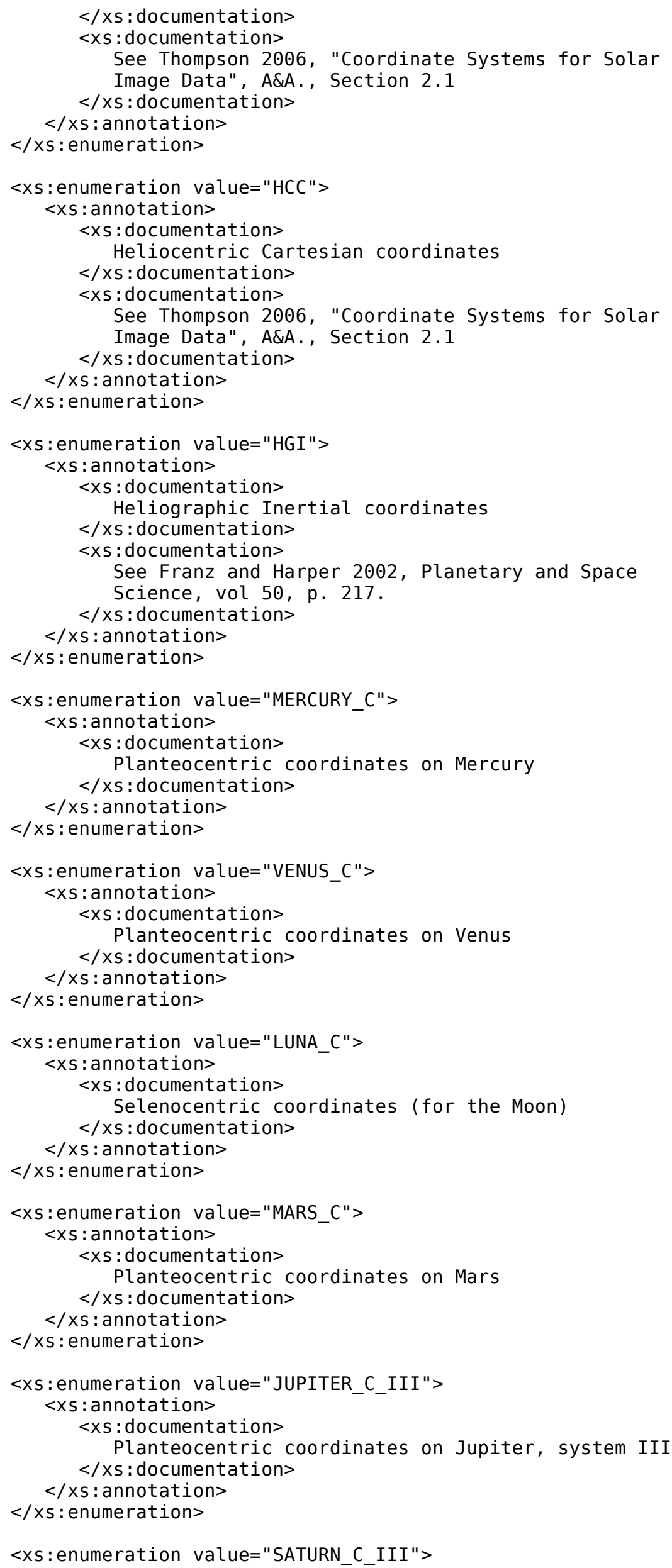




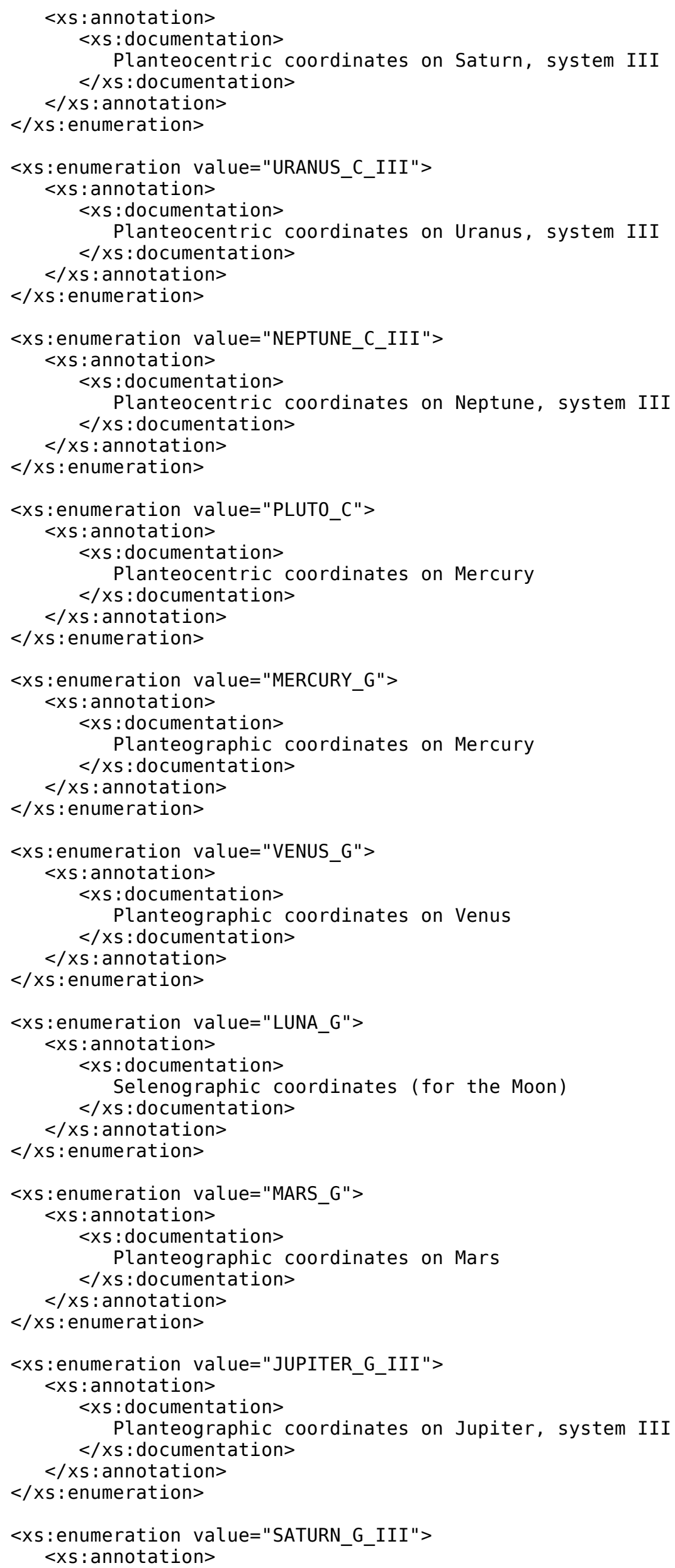




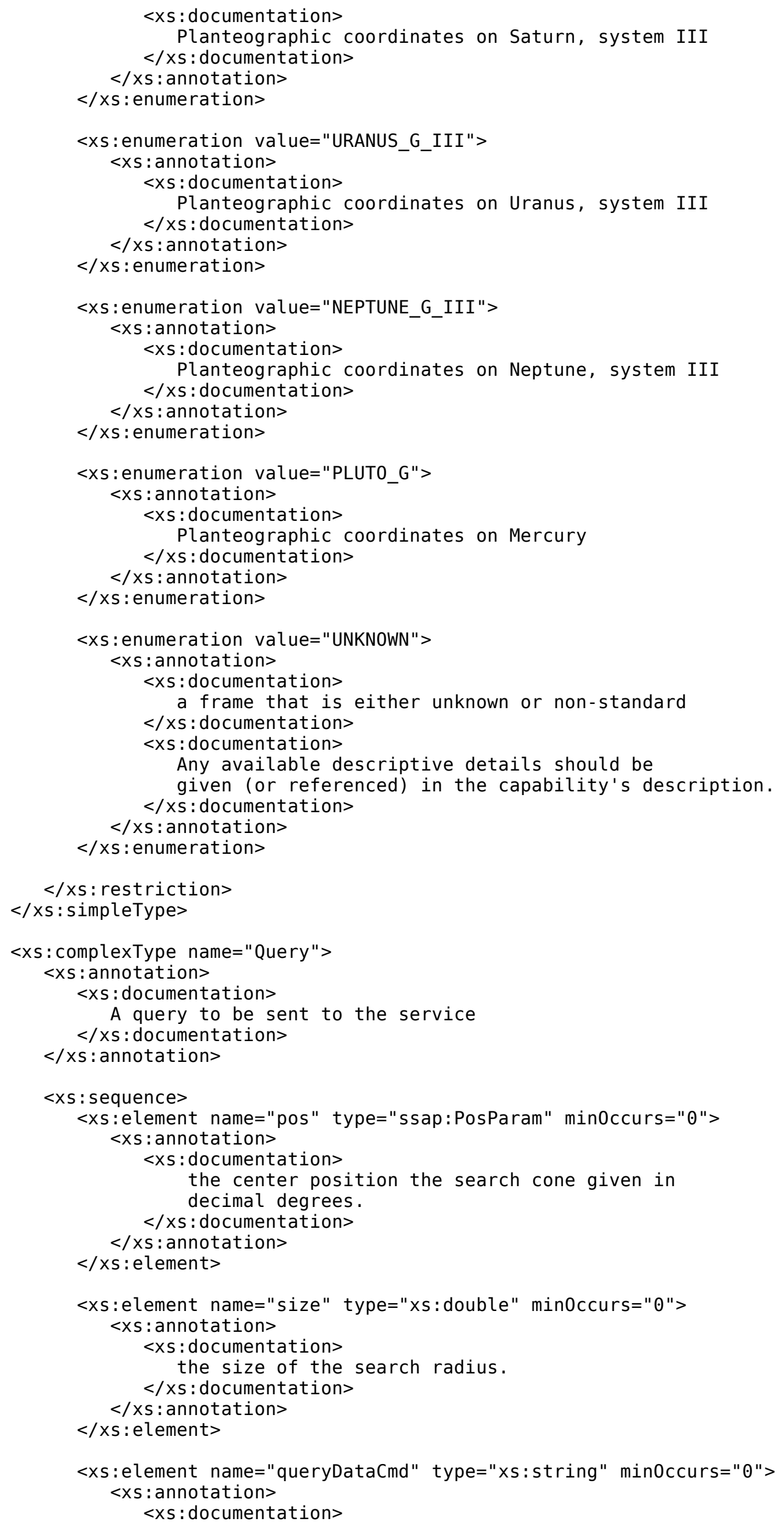


Fully specified test query formatted as an URL

argument list in the syntax specified by the SSA standard.

The list must exclude the REQUEST argument which is

assumed to be set to "queryData".

$</ x s$ : documentation $>$

$<x$ : documentation $>$

This value must be in the form of name=value

pairs delimited with apersands $(\&)$. A query

may then be formed by appending to the base URL the

request argument, "REQUEST=queryData\&", followed

by the contents of this element.

$</ x s$ : documentation $>$

$</ x s$ : annotation>

$</ x s$ : element $>$

$</ x$ : sequence $>$

$</ x s$ : complexType>

$<x s$ : complexType name="PosParam">

$<x$ : annotation>

$<x$ : documentation>

a position in the sky to search.

$</ x s$ : documentations

$</ x s$ : annotation $>$

$<x$ : sequence $>$

$<x s$ :element name="long" type="xs:double">

$<x$ : annotation>

$<x s$ : documentation>

The longitude (e.g. Right Ascension) of the center of the search position in decimal degrees.

$</ x s$ : documentation $>$

$</ x s$ : annotation $>$

$</ x s$ : element $>$

$<x s$ :element name="lat" type="xs:double">

$<x$ : annotation>

$<x s$ : documentation>

The latitude (e.g. Declination) of the center of the

search position in decimal degrees.

$</ x s$ : documentation $>$

$</ x s$ : annotation $>$

$</ x s$ : element $>$

$<x s$ : element name $="$ refframe" type $=" x s$ : token" min0ccurs $=" \Theta ">$

$<x$ : annotation>

$<x s$ : documentation>

the coordinate system reference frame name indicating

the frame to assume for the given position. If not

provided, ICRS is assumed.

$</ x s$ : documentation $>$

$</ x$ : annotation $>$

$</ x s$ : element $>$

$</ x s$ : sequence $>$

$</ x s$ : complexType>

$<!--$

- a separate type for pre-v1.0 compliant spectral services.

$-->$

<xs: complexType name="ProtoSpectralAccess">

$<x s$ : annotation>

$<x s$ : documentation>

The capabilities of an proto-SSA service implementation.

Clients may assume a particular interface for this type of service based on historical convention; however, no

guarantees are made that the service is compliant with any IVOA standard.

$</ x s$ : documentation $>$

$<x$ : documentation>

This capability is for spectral access services developed

prior to the completion of the SSA standard and,

therefore, are not compliant with that standard.

$</ x s$ : documentation $>$

$</ x$ : annotation $>$ 
$<x s$ : complexContent>

$<x$ : extension base="ssap:SSACapRestriction">

$<x s$ : sequence $>$

$<x$ :element name="dataSource" type="ssap:DataSource"

min0ccurs="1" max0ccurs="unbounded">

<xs:annotation>

$<x s$ : documentation>

The category specifying where the data originally

came from.

$</ x s$ : documentation $>$

$<x$ : documentation>

Allowed values are "survey", "pointed", "custom", "theory", "artificial"

$</ x s$ : documentation $>$

$</ x s$ : annotation $>$

$</ x s$ : element $>$

$<x$ :element name="creationType" type="ssap:CreationType"

min0ccurs $=" 1 "$ max0ccurs="unbounded" >

<xs: annotation>

$<x s$ : documentation>

The category that describes the process used to

produce the dataset.

$</ x s$ : documentation $>$

$<x s$ : documentation $>$

Typically this describes only the processing

performed by the data service, but it could

describe some additional earlier processing as

well, e.g., if data is partially precomputed.

$</ x s$ : documentation $>$

$<x s$ : documentation>

Allowed values are "archival", "cutout", "filtered"

"mosaic", "projection", "spectralExtraction",

"catalogExtraction"

$</ x s$ : documentation $>$

$</ x$ : annotation $>$

$</ x$ s: element $>$

<xs:element name="maxSearchRadius" type="xs: double" min0ccurs $=" \theta "$ max0ccurs $=" 1 ">$

$<x$ : annotation>

$<x s$ : documentation>

The largest search radius, in degrees, that will be accepted by the service without returning an error condition. Not providing this element or specifying a value of 180 indicates that there is no restriction.

$</ x s$ : documentation $>$

$<x s$ : documentation>

Not providing a value is the prefered way to

indicate that there is no restriction.

$</ x s$ : documentation $>$

$</ x s$ : annotation $>$

$</ x s$ : element $>$

$<x s$ :element name="maxRecords" type="xs:positiveInteger">

$<x$ : annotation>

$<x s$ : documentation>

The hard limit on the largest number of records that

the query operation will return in a single response

$</ x s$ : documentation $>$

$</ x$ : annotation $>$

$</ x s$ : element $>$

$<x s$ :element name="defaultMaxRecords" type="xs:positiveInteger"> $<x$ : annotation>

$<x s$ : documentation>

The largest number of records that the service will

return when the MAXREC parameter not specified

in the query input.

$</ x s$ : documentation $>$ 
$</ x s$ : annotation $>$

$</ x s$ : element $>$

$<x s$ :element name="maxAperture" type="xs:double" min0ccurs $=" \theta ">$

<xs:annotation>

$<x s$ : documentation>

The largest aperture diameter that can be supported

upon request via the APERTURE input parameter by a

service that supports the spectral extraction

creation method. A value of 360 or not providing

a value means there is no theoretical limit.

$</ x s$ : documentation $>$

$<x s$ : documentation>

Not providing a value is the preferred way to

indicate that there is no limit.

$</ x s$ : documentation $>$

$</ x s$ : annotation $>$

$</ x s$ : element $>$

$<x$ :element name="maxFileSize" type="xs:int"

minoccurs $=" \theta "$ max0ccurs $=" 1 ">$

$<x$ : annotation $>$

$<x$ : documentation $>$

The maximum image file size in bytes.

$</ x s$ : documentations

$</ x s$ : annotation $>$

$</ x s$ : element $>$

<xs:element name="testQuery" type="ssap:Query" minoccurs $=" 0 "$ max0ccurs $=" 1 ">$

$<x$ : annotation>

$<x s$ : documentation>

a set of query parameters that is expected to

produce at least one matched record which can be used to test the service.

$</ x s$ : documentation $>$

$</ x s$ : annotation $>$

$</ x s$ : element $>$

$</ x s$ : sequence $>$

$</ x$ : extension $>$

$</ x$ : complexContent $>$

$</ x s$ : complexType>

$</ x s$ : schema $>$

\section{A.4. The SLAP XML Schema}

\section{The Complete SLA Schema}

$<$ ?xml version="1.0" encoding="UTF-8"?>

<xs: schema xmlns: xs="http://WwW.w3.org/2001/XMLSchema"

xmlns: vr="http://www. ivoa. net/xml/V0Resource/v1.0"

xmlns: slap="http://www. ivoa. net/xml/SLAP/v1. $\odot$ "

xmlns: vm="http://www. ivoa. net/xml/VoMetadata/v0.1"

targetNamespace $="$ http $: / /$ www. ivoa. net/xml/SLAP/v1.0"

elementFormDefault="unqualified" attributeFormDefault="unqualified"

version=" $1.0 ">$

$$
<!--
$$

First version $09 / 09 / 09$

Authors: Jesus Salgado/Aurelien Stebe (ESAV0)

$$
-->
$$

$<x$ : annotation $>$

$<x$ : appinfo>

$<$ vm: schemaName $>$ SLAP $</ v m$ : schemaName $>$

$<$ vm: schemaPrefix $>$ xs $</ v m$ : schemaPrefix $>$

$<$ vm: targetPrefix $>$ slap</vm: targetPrefix $>$

$</ x s$ :appinfo>

$<x s$ : documentation> 
XML Schema used to describe the capabilities of a service instance conforming to the Simple Line Access Protocol (SLAP).

$</ x s$ : documentation $>$

$</ x$ : annotation $>$

<xs: import namespace="http://www. ivoa.net/xml/V0Resource/v1.0" schemaLocation="http://www. ivoa.net/xml/V0Resource/v1.0"/>

$<$ ! - - Set the Capability standardID to indicate the SLAP protocol. - - > $<x$ : complexType name="SLAPCapRestriction" abstract="true">

$<x$ : annotation>

$<x s$ : documentation>

An abstract capability that fixes the standardID to the

IVOA ID for the SLAP standard.

$</ x s$ : documentation $>$

$<x s$ : documentation>

See vr:Capability for documentation on inherited children.

$</ x s$ : documentations

$</ x s$ : annotation $>$

$<x$ : complexContent>

<xs: restriction base="vr:Capability">

$<x$ : sequence $>$

$<x s:$ element name="validationLevel" type="vr:Validation"

min0ccurs $=" \Theta "$ max0ccurs $=$ "unbounded" />

$<x$ :element name="description" type="xs:token" minoccurs $=" 0 " />$

$<x s$ :element name="interface" type="vr:Interface" min0ccurs $=" 0 "$ max0ccurs $="$ unbounded" $/>$

$</ x$ : sequence $>$

$<x s$ :attribute name="standardID" type="vr:IdentifierURI"

$</ x s$ : restriction $>$

use="required" fixed="ivo://ivoa.net/std/SLAP"/>

$</ x s$ : complexContent $>$

$</ x s$ : complexType>

$<$ ! - - SLAP Capabilities - ->

$<x s$ : complexType name="SimpleLineAccess">

$<x s$ : annotation>

$<x$ : documentation>

The capabilities of an SLAP service implementation.

$</ x s$ : documentations

$</ x s$ : annotation $>$

$<x$ : complexContent>

$<x$ : extension base="slap:SLAPCapRestriction">

$<x s$ : sequence>

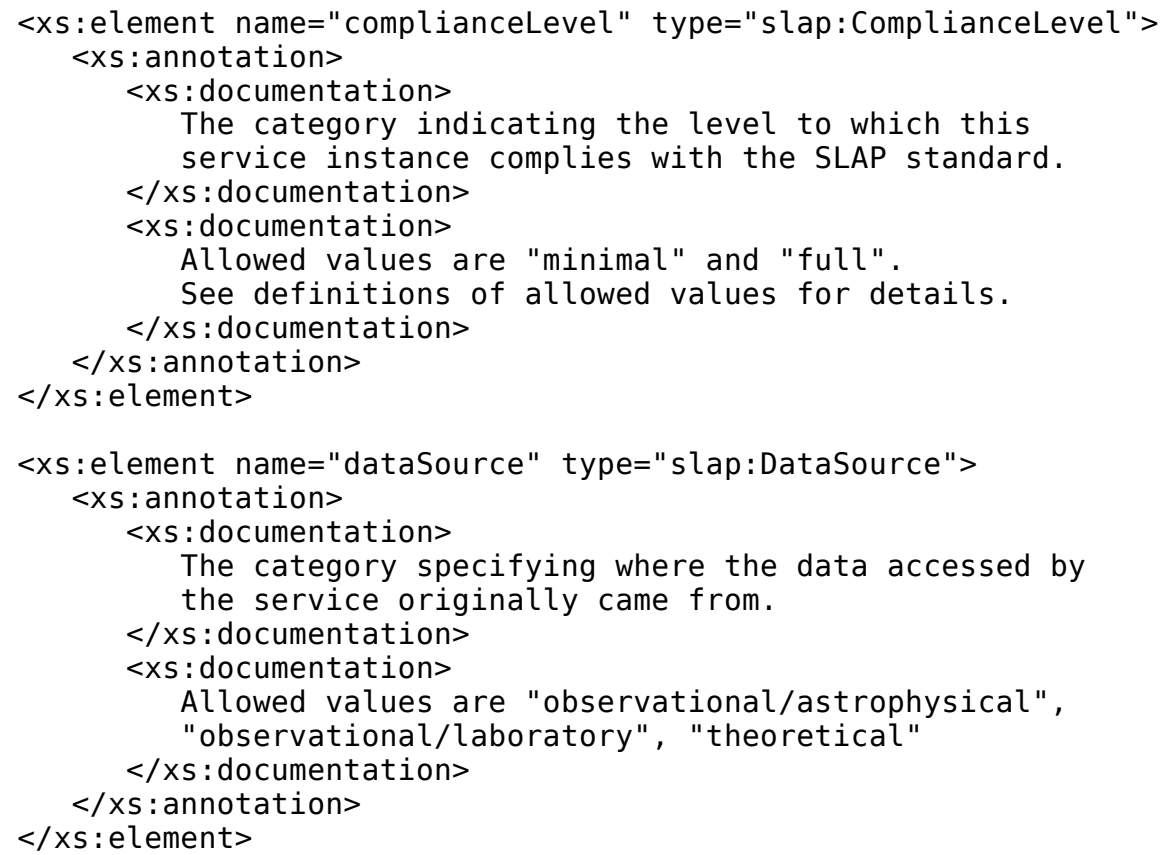




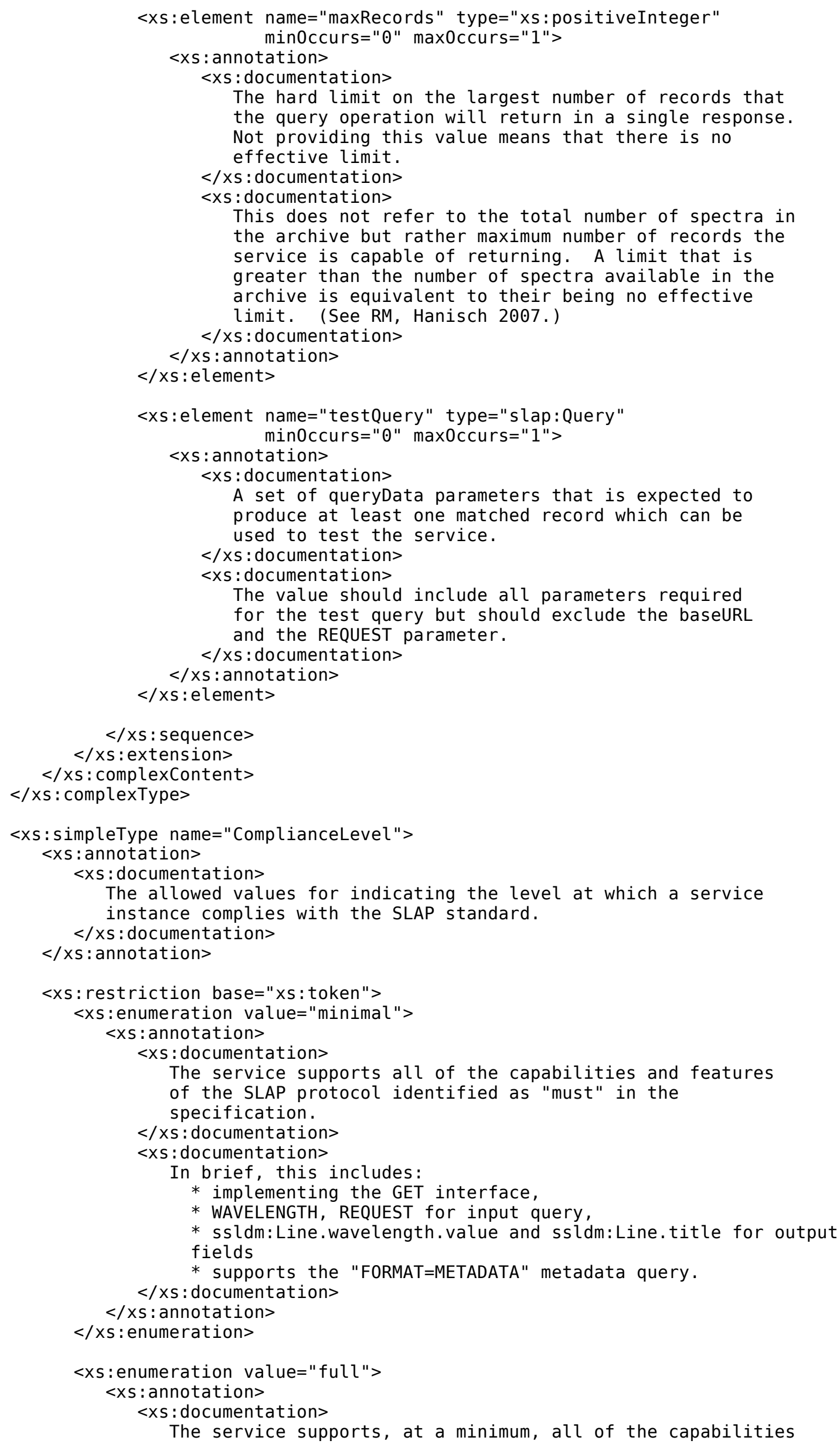


and features of the SLAP protocol identified as "must" or

"should" in the specification.

$</ x s$ : documentation $>$

$</ x s$ : annotation $>$

$</ x s$ : enumeration $>$

$</ x s$ : restriction $>$

$</ x$ : simpleType $>$

$<x$ : simpleType name="DataSource">

$<x$ : annotation>

$<x$ : documentation>

The defined categories that specify where the line data

originally came from, i.e., the type of data collections to

which the service provides access.

$</ x s$ : documentation $>$

$</ x s$ : annotation $>$

$<x s$ : restriction base $=" x s$ : token" $>$

$<x$ : enumeration value="observational/astrophysical">

$<x$ : annotation>

$<x s$ : documentation>

Lines observed and identified in real spectra of

astrophysical observations by different

instrument/projects

$</ x s$ : documentation $>$

$</ x s$ : annotation $>$

$</ x s$ : enumeration $>$

$<x$ : enumeration value="observational/laboratory">

$<x s$ : annotation>

$<x s$ : documentation>

Lines observed and identified in real spectra of

laboratory measurements

$</ x s$ : documentation>

$</ x s$ : annotation $>$

$</ x s$ : enumeration $>$

<xs: enumeration value="theoretical ">

$<x$ : annotation>

$<x$ : documentation>

Servers containing theoretical spectral lines

$</ x s$ : documentations

$</ x$ : annotation $>$

$</ x s$ : enumeration $>$

$</ x s$ : restriction $>$

$</ x s$ : simpleType>

$<x s$ : complexType name="Query">

$<x$ : annotation>

$<x s$ : documentation>

A query to be sent to the service, e.g., a test query.

$</ x s$ : documentation $>$

$</ x s$ : annotation $>$

$<x s$ : sequence>

<xs:element name="wavelength" type="slap:WavelengthRange" min0ccurs=" $\odot ">$

$<x s$ : annotation>

$<x s$ : documentation>

Spectral range in meters to be used to constrain the query of spectral lines.

$</ x s$ : documentation $>$

$</ x s$ : annotation $>$

$</ x s$ : element $>$

<xs:element name="queryDataCmd" type="xs:string" min0ccurs=" $\odot$ "> $<x$ : annotation>

$<x$ : documentation>

Fully specified queryData test query formatted as an URL argument list in the syntax specified by the SLAP standard. The list must exclude the REQUEST argument which is assumed to be set to "queryData". VERSION may be included if the test query applies to a specific version 
of the service protocol.

$</ x s$ : documentation $>$

$<x s$ : documentation>

If queryDataCmd is used to form a query, the default

value of WAVELENGTH specified above is not

used; if the test query requires WAVELENGTH it

should be included directly in queryDataCmd.

$</ x s$ : documentation $>$

$<x s$ : documentation>

This value must be a string in the form of name=value

pairs delimited with ampersands $(\&)$. A query may

then be formed by appending to the baseURL the request

argument, "REQUEST=queryData\&", followed by the

contents of this element.

$</ x s$ : documentation $>$

$</ x$ : annotation $>$

$</ x s$ : element $>$

$</ x$ : sequence $>$

$</ x s$ : complexType>

<xs: complexType name="WavelengthRange" >

$<x$ : annotation>

$<x s$ : documentation>

Spectral range in meters to be used to constrain the query

of spectral lines

$</ x s$ : documentation $>$

$</ x s$ : annotation $>$

$<x s$ : sequence>

$<x s$ : element name="minWavelength" type="xs:double" min0ccurs $=" \odot ">$

$<x$ : annotation>

$<x s$ : documentation>

Minimum wavelength in meters to be used to constrain the query

of spectral lines

$</ x s$ : documentations

$</ x s$ : annotation $>$

$</ x$ s: element $>$

$<x s$ :element name="maxWavelength" type="xs:double" min0ccurs=" $\odot ">$

$<x$ : annotation>

$<x s$ : documentation>

Maximum wavelength in meters to be used to constrain the query

of spectral lines

$</ x s$ : documentations

$</ x s$ : annotation $>$

$</ x s$ : element $>$

$</ x s$ : sequence $>$

$</ x s$ : complexType>

$</ x s$ : schema $>$

\section{Appendix B: Supporting Multiple Versions of DAL Protocols}

Note: this section is non-normative.

It is possible for a VOResource-encoded resource description to indicate support for multiple versions of standard service. This is described generally in Section 2.2.2 ("The Service Data Model") of the VOResource specification [VOR]. In that section, the specification says that a <capability> element can contain multiple <interface> elements, each describing a different version. In this appendix, we illustrate how this can be applied to the DAL services covered by this (SimpleDALRegExt) specification.

We start by noting that the standardID values for each of the DAL protocols described in this document refer to the standards generally, without reference to the particular version. For example, the IVOA identifier for the Simple Cone Search protocol is ivo://ivoa. net/std/ConeSearch. Thus a <capability> element can logically describe support for any version or multiple versions of the standard DAL protocol as long as the extension schema for that protocol is same for all of the versions.

Here is an example a service that supports two versions of the SSA protocol: 


\section{Example}

Supporting multiple versions of the SSA standard. Below shows just the <capability> element of a description of an SSA service resource.

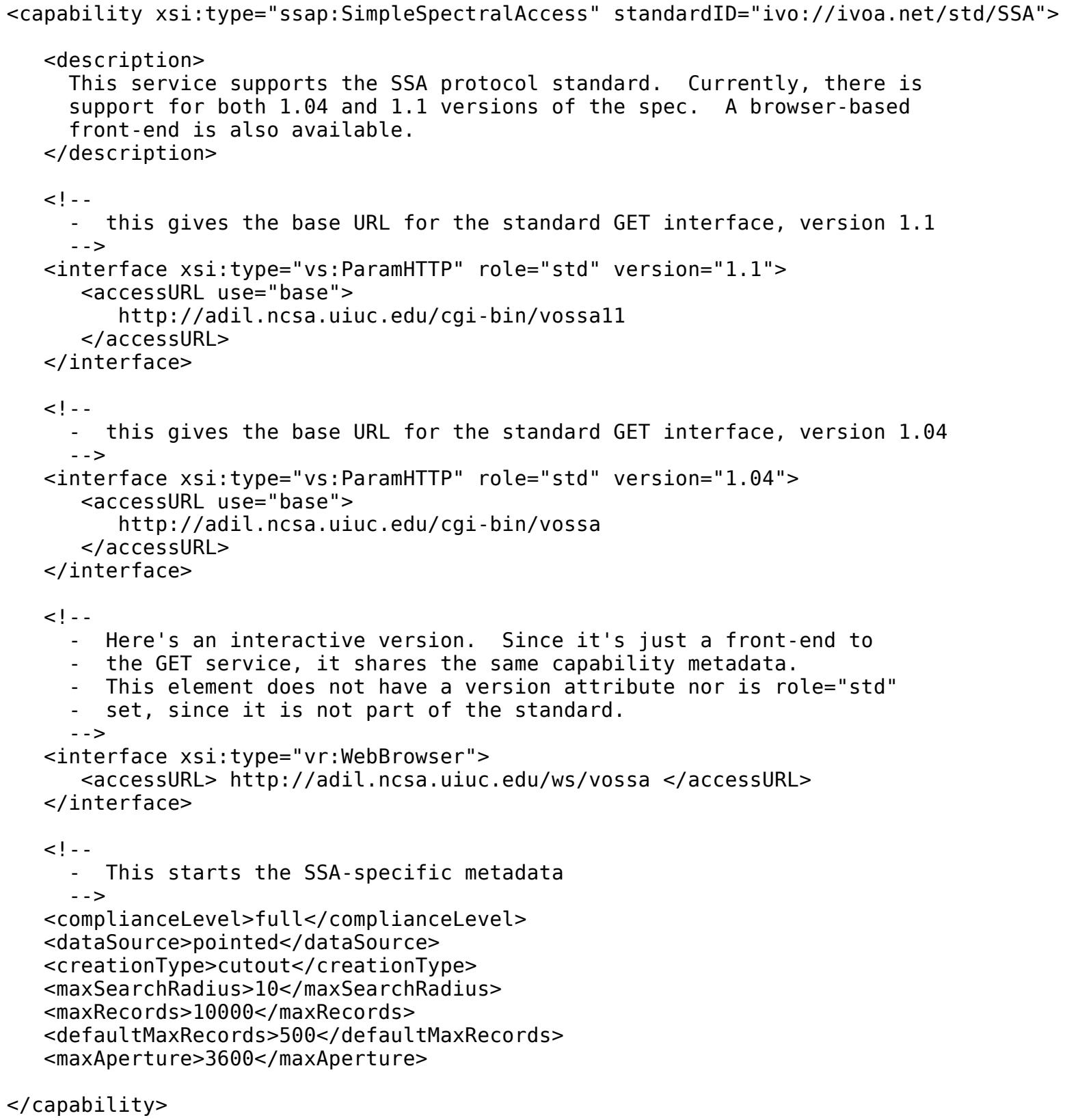

In the above example, two different SSA service endpoints are supported, each compliant with a different version of the SSA standard. The version attribute on the <interface> elements indicate which version the endpoint supports. Note that if a version attribute is not provided, it defaults to "1.0". The role="std" distinguishes standard-complying interfaces from custom ones; in the example above, an endpoint to a browser-based front-end is provided as well.

A consumer that wishes to engage a standard DAL service, should extract interface descriptions where role="std" is set. From of those, the consumer should inspect the version attribute to select a version that it supports.

Often, the difference between versions is of little consequence to a consumer, so having to choose between several standardized versions is an unneeded complication. Thus, it is recommended that publishers list the most preferred version for users (typically the latest) first among the list of interfaces; consequently, consumers that do not care to attempt to interpret the version attribute should choose the first in the list. If the service query fails (because the consumer's assumptions about the service protocol are not applicable to that version of the service), the consumer may try the next one in the list. 


\section{Changes since PR-v1.0 20130911:}

- none other than date and status.

\section{Changes from PR-v1.0 20121116}

- for SSA's creationType, changed specialExtraction to spectralExtraction.

- corrected Creation Type reference to section in SSA doc.

- made long and lat elements in ssap : PosParam required.

- incremented SSA schema version to 1.1 in namespace.

- refresh App. A from official schemas

- fixed typos ("IRCS" and value type for maxFilesize)

- noted that the <long $>$ and <lat $>$ values within the sia: SkySize type are given in degrees.

- Fixed documentation of SIA's sia: Query type in the schema.

\section{Changes from PR-v1.0 20120517}

- The namespace URIs given in Sections 3.1.1, 3.2.1, 3.3.1, and 3.4.1 were updated to match that specified in the XSDs (i.e. to include a "v" preceding the version field).

- Several capability metadata with types xs : int and xs : float were changed to xs : positiveInterger xs : double to allow for larger/more precise numbers.

- Capability metadata that indicated maximum allowed values (e.g. <maxRecords>, <maxImageSize>, etc.) were made optional to avoid large, meaningless numbers from being provided. Now not specifying a value is the preferred way to indicate that no upper limit applies.

- Semantic definition of <sia : maxImageExtent> clarified to differentiate it from <sia : maxQueryRegionSize>

- The type for <sia: maxImageSize> was changed to xs : positiveInteger, a single number that represents the length of a side in pixels. The sia: ImageSize type (no longer needed) was dropped.

- The version field in the SIA namespace was incremented to 1.1 due to the non-backward-compatible change to <sia : maxImageSize>

- various typos and grammatical errors corrected.

\section{Changes from WD-v1.0 20110921:}

- Now recommend ssap as prefix; changed all occurances of ssa in text and schema.

- added <supportedFrame> to ssap: SimpleSpectralAccess

- removed import of VODataService schema from SIA, SSA, and Conesearch schemas.

- change base type of controlled vocab types from xs : string to xs : token for consistancy with VOResource.

\section{References}

\section{[RFC 2119]}

Bradner, S. 1997. Key words for use in RFCs to Indicate Requirement Levels, IETF RFC 2119, [schema] http: //www. ietf.org/rfc/rfc2119.txt

Fallside, David C., Walmsley, Priscilla (editors) 2004, XML Schema Part 0: Primer Second Edition, [Arch] W3C Recommendation 28 October 2004, http: //www.w3.org/TR/xmlschema-0/

Arviset, Christophe and the IVOA Tehcnical Coordination Group 2010, The IVOA in 2010: Technical Assessment and Roadmap, v1.0, IVOA Note, in preparation. [SCS]

Williams, R., Hanisch, R., Szalay, A., and Plante, R. 2008, Simple Cone Search Version 1.03, IVOA Recommendation, 22 February 2008, http: //Www. ivoa. net/Documents/REC/DAL/ConeSearch 20080222 . html. 
[SIA]

Tody, D. and Plante, R. 2004, Simple Image Access Specification Version 1.0, IVOA Working Draft, http : //WWw . ivoa . net/Documents/WD/SIA/sia-20040524 . html.

[SSA]

Tody, D., Dolensky, M., McDowell, J., Bonnarel, F., Budavari, T., Busko, I., Micol, A., Osuna, P, Salgado, J., Skoda, P., Thompson, R., and Valdes, F. 2008, Simple Spectral Access Protoco/ Version 1.04, IVOA Recommendation, 01 Febrary 2008, http: //wWw. ivoa. net/Documents/cover/SSA20080201. html.

[SLA]

Salgado, J., Osuna, P., Guainazzi, M., Barbarisi, I., Dubernet, M., and Tody. D. 2010, Simple Line Access Protoco/ Version 1.0, IVOA Recommendation, 9 December 2010,

http : //WwW . ivoa. net/Documents/SLAP/20101209/.

[RI]

Benson, Kevin, Plante, Ray, Auden, Elizabeth, Graham, Matthew, Greene, Gretchen, Hill, Martin, Linde, Tony, Morris, Dave, O'Mullane, Wil, Rixon, Guy, Andrews, Kona 2008, IVOA Registry Interfaces, v1.02, IVOA Recommendation, http: //www. ivoa. net/Documents/latest/ResourceInterface.html

[RM]

Hanisch, Robert (ed.) 2004. Resource Metadata for the Virtual Observatory, Version 1.12, IVOA

Recommendation, http: //www . ivoa. net/Documents/REC/ResMetadata/RM-20040426. htm [VOR]

Plante, R., Benson, K., Graham, M., Greene, G., Harrison, P., Lemson, G., Linde, T., Rixon, G., Stébé, A. 2008, VOResource: an XML Encoding Schema for Resource Metadata, v1.03, IVOA

Recommendation, http: //www. ivoa . net/Documents/REC/ReR/VOResource-20080222. html [VDS]

Plante, R., Stébé, A. Benson, K., Dowler, P. Graham, M., Greene, G., Harrison, P., Lemson, G., Linde, T., Rixon, G., Stébé, A. 2009, VOData Service: a VOResource Schema Extension for Describing Collections and Services, v1.1, IVOA Recommendation, http: //www . ivoa . net/Documents/V0DataService/20101202/

[STC]

Rots, Arnold 2007, Space-Time Coordinate Metadata for the Virtual Observatory, v1.33, IVOA Recommendation, href="http : //www . ivoa . net/Documents/REC/STC/STC-20071030. html"> 